\title{
Comment les omnipraticiens expliquent-ils leurs pratiques tarifaires? Quelques paradoxes de la politique de santé
}

How the general practitioners explain their pricing practices? Some paradoxes of the health policy

Philippe Abecassis et Jean-Paul Domin

\section{OpenEdition \\ Journals}

Édition électronique

URL : http://journals.openedition.org/pmp/4137

ISSN : 2119-4831

Éditeur

Institut de Management Public (IDPM)

\section{Édition imprimée}

Date de publication : 15 mars 2011

ISSN : 0758-1726

Référence électronique

Philippe Abecassis et Jean-Paul Domin, « Comment les omnipraticiens expliquent-ils leurs pratiques tarifaires? Quelques paradoxes de la politique de santé », Politiques et management public [En ligne], Vol 28/1 | 2011, mis en ligne le 01 octobre 2012, consulté le 19 avril 2019. URL : http:// journals.openedition.org/pmp/4137 


\title{
Comment les omnipraticiens expliquent-ils leurs pratiques tarifaires? Quelques paradoxes de la politique de santé
}

\author{
Philippe Abecassis ${ }^{a}$ et Jean-Paul Domin ${ }^{b *}$ \\ ${ }^{a}$ CEPN, Université Paris Nord - ${ }^{b}$ OMI, Université de Reims Champagne-Ardenne
}

\section{Résumé}

Ce travail propose une réflexion sur les effets auprès des médecins de l'acharnement des politiques publiques à limiter la dérive des dépenses de santé en se fondant sur le seul paradigme marchand. Cette politique incitative, qui prend appui sur la rationalité stratégique des agents, a des effets assez paradoxaux dans la mesure où, en souhaitant maîtriser les dépenses, elle active des comportements dépensiers. Après avoir développé l'hypothèse théorique et présenté les méthodes utilisées et les spécificités de l'échantillon étudié, l'article analyse le discours des praticiens sur des points importants concernant certaines de leurs pratiques tarifaires, notamment en matière de dépassements d'honoraires et d'actes gratuits.

(c) 2011 IDMP/Lavoisier SAS. Tous droits réservés

Mots clés : actes gratuits, dépassements d'honoraires, incitations, médecine ambulatoire, politique économique de santé.

\section{Abstract}

How the general practitioners explain their pricing practices? Some paradoxes of the health policy: This study looks at the effects on doctors of policy-makers'obsession with cutting health costs by focusing purely on commercial criteria. This incentive-based policy - which relies on agents'strategic rationality - has somewhat paradoxical effects in so much as, in endeavoring to control spending, it actually encourages overspending modes of behavior. After setting out a theoretical hypothesis and presenting the methods used as well as the specific character of the sample studied, the paper analyzes practitioners' statements on the matter and some of their pricing practices, notably overcharging and free care. @ 2011 IDMP/Lavoisier SAS. Tous droits réservés

Keywords: free care, overcharging, incentives, primary care, health policy.

\footnotetext{
*Auteur correspondant : jp.domin@univ-reims.fr doi:10.3166/pmp.28.27-55 @ 2011 IDMP/Lavoisier SAS. Tous droits réservés
} 


\section{Introduction}

Le système français d'assurance-maladie repose sur une organisation complexe. Les services de soins sont fournis par des prestataires publics (hôpitaux) et privés (cliniques et médecine ambulatoire). Les patients peuvent choisir librement leurs praticiens en payant les services à l'acte. Depuis 1971, les honoraires du corps médical sont définis par des conventions signées par les représentants de la profession et des caisses d'assurance-maladie. Jusqu'au début des années 1970, le système a fonctionné à guichet ouvert, c'est-à-dire sans limitation des dépenses (Palier B., 2005).

Les politiques de freinage des dépenses et de diminution des coûts sont mises en œuvre depuis le milieu des années 1970. Elles cö̈ncident avec un changement de référentiel de politiques publiques, mais se déclinent de façon différente suivant les périodes (Théret B., 2007). Dans un premier temps, les pouvoirs publics ont favorisé des mesures centrées sur l'offre. Durant la phase de stagflation, (1975-1983), un dispositif de limitation du nombre de diplômes délivrés (numerus clausus), institué en 1971, est renforcé. Il a pour objectif de réduire l'offre de soins censée participer à la croissance de la demande. La période de désinflation compétitive (1983-1993) marque une certaine continuité politique (resserrement du numerus clausus, mise en place des taux directeurs et du budget global...). Elle est complétée par une tentative de désocialisation de la demande de soins (développement d'un secteur 2 dit à honoraires libres). Mais ces mesures se révèlent vite financièrement inefficaces et socialement inégalitaires.

Un premier revirement a lieu au milieu des années 1990 avec une forte affirmation des préférences béveridgiennes des élites (renforcement du poids décisionnel législatif, fiscalisation du financement, réforme de l'offre publique de soins...). Dans le même temps, la tutelle abandonne toute tentative de réforme de l'offre privée de soins qui lui paraît trop risquée au plan politique. Depuis le début des années 2000, la politique économique de santé prône une régulation par la demande. La théorie économique des anticipations rationnelles, en soutenant que les mesures keynésiennes sont non seulement stériles mais également nuisibles pour l'économie capitaliste, a contribué à cette évolution. Désormais, la politique économique de santé repose sur un système complexe d'incitations censées orienter le comportement des individus. C'est dans ce sens qu'il faut y voir une évolution marchande (Batifoulier P., Domin J.-P. et Gadreau M., 2007a).

La politique économique de santé modifie les comportements du corps médical, c'est son but. Mais au-delà des comportements, les représentations et les discours du corps médical sont remodelés par « l'univers marchand » sur lequel s'appuient ces politiques. En s'appuyant sur cette hypothèse, ce travail propose une réflexion sur les relations entre les comportements tarifaires des médecins et les mécanismes incitatifs sur lesquels s'appuie la politique de santé. L'hypothèse retenue est que les agents répondent par des comportements marchands aux nombreuses politiques publiques visant à limiter la dérive des dépenses de santé en se fondant sur le seul paradigme du marché. La persistance d'un déficit chronique est, en effet, souvent associée aux comportements irresponsables des assurés sociaux (thèse de l'aléa moral) et la politique économique de santé a tenté d'y répondre en mettant en place un dispositif d'incitations. Or, cette politique incitative qui prend appui sur la rationalité stratégique des agents a des effets assez paradoxaux dans la mesure où, en souhaitant maîtriser les dépenses, elle peut activer des comportements dépensiers (Batifoulier P., Gadreau M. et Lievaut J., 2009). 
Nous organiserons notre propos en deux temps. Après avoir développé l'hypothèse théorique, nous présenterons les méthodes utilisées et les spécificités de l'effectif $(\S 1)$. Nous analyserons ensuite le discours de ces praticiens sur des points importants concernant certaines de leurs pratiques tarifaires, notamment en matière de dépassements d'honoraires et d'actes gratuits $(\S 2)$.

\section{Politique économique de santé et pratiques tarifaires : cadre théorique et méthodologie}

Ce travail s'inscrit dans la continuité d'un rapport remis à la DREES-MiRe et analysant l'incapacité de la politique de santé à contrecarrer la dérive des dépenses de santé (Batifoulier P., Gadreau M. et Vacarie I., 2008). L'objectif est d'éclairer l'impact de la politique économique de santé sur la pratique tarifaire des acteurs en se fondant sur les explications fournies par les acteurs. Après avoir présenté le cadre théorique de ce travail $(\S 1.1)$, nous présenterons les méthodes utilisées et les spécificités de l’effectif (§1.2).

\subsection{Politique économique de santé et discours des acteurs}

Si la politique économique de santé a privilégié jusque dans les années 1990 une régulation sur l'offre, à partir de cette date, le référentiel a évolué et, avec lui, la façon d'appréhender les réformes du système de soins. L'évolution de la politique économique de santé depuis cette date (§1.1.1.) a accéléré une reconfiguration du discours des acteurs (§1.1.2.).

\subsubsection{La politique économique de santé comme moteur des transformations de ce secteur}

Depuis le début des années 1990 et le renouvellement de l'analyse des politiques publiques en France, la notion de référentiel tient une place croissante dans l'analyse des politiques publiques. Bien qu'abondamment controversée ${ }^{1}$, la notion de référentiel a l'avantage de proposer une analyse des processus de construction des catégories qui fondent l'action publique. En s'appuyant sur la dimension cognitive de l'action publique, cette analyse se fixe pour objectif de rendre compte de la pluralité des logiques d'action mises en œuvre. Dans ce cadre, c'est toujours en s'appuyant sur un régime d'idées spécifique ${ }^{2}$ que les acteurs organisent les mesures qu'ils souhaitent prendre. Le référentiel correspond, selon Pierre Muller, «avant tout à une certaine vision de la place et du rôle du secteur concerné dans la société [...] le référentiel d'une politique est constitué d'un ensemble de prescriptions qui donne du sens à un programme politique en définissant des critères de choix et des modes de désignation des objectifs » (Muller P., 2003).

Le référentiel articule quatre niveaux de perception du monde qui sont les valeurs, les normes, les algorithmes et les images. Les premières correspondent à des représentations fondamentales du monde qui doivent définir un cadre global pour l'action publique. Les normes définissent les écarts entre le réel perçu et le réel souhaité par les acteurs et servent de socle aux principes d'action (Muller P., 2000). Les algorithmes développent des instru-

\footnotetext{
1 Voir à ce propos: (Faure A., Pollet G. et Warin P., 1995), (Leca J. et al., 1996) ou (Hassenteufel P. et Smith A., 2002).

${ }^{2}$ Le « régime des idées » est à la base de la notion de paradigme (Hall P. A., 1993) ou de référentiel (Muller P., 2000).
} 
ments et les images constituent des vecteurs implicites des valeurs : le «trou de la sécu » et le malade fraudeur en sont des exemples significatifs.

Alors que le référentiel keynésien a pendant longtemps façonné l'État social en légitimant des politiques sociales appréhendées comme un soutien de la demande effective. Les années 1990 marquent un tournant. Dorénavant l'objectif est de réduire les dépenses sociales afin de réduire les charges des entreprises (Palier B., 2008). L'État social évolue désormais en s'appuyant de façon croissante sur un référentiel marchand qui renvoie explicitement à l'efficacité des mécanismes de marché et s'oppose à l'intervention de l'État (Batifoulier P., Domin J.-P. et Gadreau M., 2007b). Le référentiel permet dans cette configuration de fournir un cadre d'interprétation du monde associant les bonnes idées aux bonnes politiques.

En matière de politique économique de santé, la théorie des incitations fournit un cadre d'interprétation où l'agent, parce qu'il est rationnel et potentiellement malhonnête, peut profiter de l'avantage informationnel dont il bénéficie et en tirer parti à des fins personnelles. La théorie de l'aléa moral, empruntée à l'économie de l'assurance, considère le patient assuré social comme étant susceptible de profiter de sa situation pour augmenter de façon injustifiée sa consommation médicale (aléa moral de seconde espèce). La seule façon de contrecarrer ces comportements consiste à définir un certain type d'incitation censé suspendre l'opportunisme de l'agent (Rochaix L., 1997).

La politique économique de santé contribue à l'activation d'un référentiel qui accorde une grande importance au marché tout en reconnaissant le rôle protecteur de l'État lorsqu'il s'appuie sur une logique de contractualisation. C'est aussi sur ces fondements, en partie via la politique de santé, que les individus s'appuient pour interpréter les règles. Le référentiel a alors une influence sur l'éthique médicale qui modifie, chez les praticiens, le comportement jugé légitime (Batifoulier P. et Gadreau M., 2006a). Des comportements nouveaux apparaissent, notamment en médecine ambulatoire, jugés conformes à l'éthique alors qu'ils favorisent la croissance des dépenses de santé. Ainsi, cette dernière serait-elle en partie endogène à la politique économique (Batifoulier P., Domin J.-P. et Gadreau M., 2007b). Deux exemples nous paraissent assez significatifs de cette évolution des pratiques : la généralisation des dépassements d'honoraires et l'érosion croissante de du nombre d'actes gratuits réalisés.

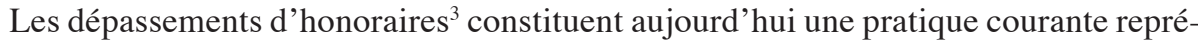
sentant une part non négligeable des revenus des praticiens : 5,5\% des honoraires totaux des omnipraticiens et 14,4\% de certains spécialistes en 2004 (Legendre N., 2006). La liberté tarifaire, qui est une revendication d'une frange du corps médical depuis le début des années 1980, légitime le dépassement d'honoraire qui est appréhendé comme le résultat d'une juste rétribution du praticien. L'érosion de la part gratuite, qui se traduit dans les faits par le caractère payant d'actes qui étaient auparavant gratuits (renouvellement d'ordonnance, vaccination, actes récurrents...) participe d'un même mouvement de marchandisation de la médecine légitimant des pratiques jugées, il y a encore quelques années, comme incorrectes (Batifoulier P. et Ventelou B., 2003)

\footnotetext{
${ }^{3}$ Le mode de rémunération des médecins français repose historiquement sur le paiement à l'acte. Tout acte médical référencé dans la nomenclature est rémunéré sous forme d'honoraires. La pratique du dépassement est liée au secteur d'appartenance du praticien. Si les médecins exerçant en secteur à honoraires différents (secteur 2) peuvent choisir librement leurs honoraires, ceux du secteur conventionné (secteur 1) doivent pratiquer les tarifs conventionnels, sauf exigence particulière du patient (supplément nuit, supplément week-end, supplément nourrisson, ...).
} 


\subsubsection{Discours des acteurs et construction des politiques de santé}

Le dispositif conventionnel régit le cadre d'activité et les honoraires des praticiens. C'est un texte discuté et validé par des représentants du corps médical et des tutelles, il constitue un compromis entre les représentations que les acteurs se font d'un monde commun (Boltanski L. et Thévenot L., 1991), mais dont les différences se repèrent à travers plusieurs espaces discursifs. Une analyse textuelle diachronique précédente, menée avec le logiciel Prospéro sur l'ensemble des conventions médicales ${ }^{4}$ et de leurs avenants de 1971 à 2005 (Abecassis P. et Domin J.-P., 2008; Abecassis P. et Domin J.-P., 2009) nous a permis de mettre en évidence l'existence de trois espaces discursifs distinctifs. De façon schématique, ces trois espaces s'organisent de la façon suivante. Le premier, l'espace discursif libéral, se scinde en un discours libéral traditionnel et un discours libéral renouvelé. Le deuxième, l'espace discursif gestionnaire relève de la tutelle et des pouvoirs publics et invoque des préoccupations d'ordre comptable. Il est peu présent dans le cadre de cette étude. Enfin, le discours marchand provient d'une fraction du corps médical qui entend légitimer la marchandisation de pratiques qui, autrefois, étaient gratuites.

L'espace discursif libéral traditionnel s'articule essentiellement, depuis le début du XIX ${ }^{\mathrm{e}}$ siècle, autour d'une critique de la médecine salariée qui a participé à la construction de l'identité professionnelle des médecins libéraux (Steffen M., 1987). L'évolution de la pratique libérale doit donc être appréhendée à l'aune de la menace du salariat et du tiers payant. Mais aussi paradoxal que cela puisse paraître, le conventionnement a permis à la profession d'évacuer les différentes formes de concurrence, notamment celle des centres de santé.

L'identité libérale se manifeste par l'indépendance, un trait caractéristique de la profession qui, par nature, s'oppose au salariat. Lier l'indépendance du praticien au caractère libéral de sa profession consiste à mettre l'accent à la fois sur une certaine forme d'autonomie intellectuelle mais également sur la liberté professionnelle. L'autonomie est un trait que partage la profession avec les magistrats ou les universitaires, mais elle ne peut pas pour autant être assimilée à une profession strictement intellectuelle. En effet, le médecin doit être appréhendé comme un travailleur intellectuel indépendant à revenus variables. Une profession libérale est donc une profession « chargée de valeurs intellectuelles ou morales qui s'exerce dans le cadre d'une petite entreprise libérale » (Hatzfeld H., 1963). Ce trait la rapproche du métier de commerçant avec un patron responsable de sa petite entreprise (Jaunait A., 2005).

L'espace discursif libéral renouvelé se structure au milieu des années 1980 autour d'une organisation syndicale : MG France. Ce discours correspond au concept de critique artiste (Boltanski L. et Chiapello É., 1999). Le médecin et l'artiste ont en commun un droit de propriété sur leur production. Le praticien est responsable de la guérison du malade et agit en son nom. Il rejette la massification et la taylorisation de la médecine au profit d'une recherche de l'authenticité. MG France s'appuie sur une nouvelle interprétation de l'éthique,

\footnotetext{
${ }^{4}$ Le système de santé, notamment la médecine ambulatoire, est depuis les années 1960 encadré par un dispositif conventionnel promulgué par décret et signé par les pouvoirs publics, les organisations syndicales représentatives de praticiens et les caisses d'assurance-maladie. L'objectif est d'attirer les professionnels dans un cadre juridique prévoyant des tarifs opposables afin d'offrir un niveau optimal de remboursement aux assurés sociaux. La politique conventionnelle tente donc de concilier deux objectifs contradictoires : la prise en charge collective de la santé dans le respect des règles de la médecine libérale.
} 
non spécifiquement associée à la médecine libérale et valorisant des formes collectives d'exercice (Robelet M., 2005). Ce syndicat défend de nouveaux principes d'organisation de la pratique (réseaux...), mais également de nouvelles formes de rémunérations (salaires, forfait, capitation...). Ce sont d'ailleurs des arguments éthiques qui les justifient : plus grande proximité avec les patients, souci de la qualité. Ce dernier argument est plus particulièrement intéressant dans la mesure où ce type de rémunération n'incite pas le praticien à accroître le nombre de ses actes, mais plutôt à se concentrer sur la qualité.

L'option « médecin référent » défendue par MG France dans le cadre de la convention de 1997 est tout à fait caractéristique du discours libéral renouvelé dont cette organisation syndicale est porteuse. Il s'agit d'une diversification de l'offre de soins qui peut signifier une acceptation de la concurrence. En effet, l'organisation prônée par MG France s'articule autour de petites unités de soins, organisées en filière et en situation de concurrence. L'option «médecin référent» doit donc être appréhendée comme «l'expression d'une identité libérale renouvelée reposant sur le modèle de la petite entreprise » (Robelet M., 2005).

Le discours marchand constitue le dernier espace discursif étudié. La référence au marchand ne constitue pas une nouveauté au sein de la profession. À la fin du XIX ${ }^{\mathrm{e}}$ siècle, le discours s'est structuré en opposition face aux premières lois sociales (loi du 15 juillet 1893 sur l'assistance médicale gratuite, Charte de la mutualité du $1^{\text {er }}$ avril $1898 \ldots$...). L'argument défendu reposait sur un refus de la socialisation qui ne serait pas en mesure, selon ses partisans, de satisfaire la demande de soins. Le médecin n'aurait donc aucun intérêt à soigner le pauvre puisqu'il ne recevrait en contrepartie qu'une indemnité sans commune mesure avec celle qu'il aurait reçue si le jeu de la concurrence n'avait pas été faussé par l'intervention publique (Domin J.-P., 2003).

Aujourd'hui, le dépassement d'honoraire constitue un exemple éloquent de discours marchand. La critique des médecins à l'encontre de l'étatisation du système de santé prend la forme de ce dépassement qui est une lutte contre le carcan tarifaire (Batifoulier P., Domin J.-P. et Gadreau M., 2007b). Pour la profession, le praticien qui choisit le secteur à honoraires libres n'affiche pas pour autant un goût pour le lucre, qui est un sujet tabou (Bloy G., 2002), mais sa préférence pour une médecine de qualité (dont la durée de consultation par exemple, est plus longue). Le dépassement d'honoraire ne s'inscrit donc pas, dans les canons de la profession, dans la perspective d'un discours marchand, celui-ci se manifeste cependant par une augmentation importante des cas de dépassements d'honoraires ainsi que par le développement de plusieurs catégories d'honoraires particuliers, rémunérant des tâches autrefois gratuites. Ces « honoraires techniques » seraient la marque de la marchandisation croissante de la médecine.

Si la tutelle s'est d'abord opposée au développement de cette logique marchande, elle applique désormais des politiques qui relèvent de celle-ci.

\subsection{Une étude statistique et lexicométrique}

Ce travail prend appui sur un échantillon de 596 médecins généralistes ayant répondu à un questionnaire. Il combine une étude statistique et une analyse lexicométrique (\$1.1.1). Les caractéristiques générales de l'effectif sont comparables à celles issues des différentes études menées sur cette population (\$1.1.2). 


\subsubsection{L'échantillon utilisé et la méthodologie de l'enquête}

L'étude porte sur une population d'omnipraticiens contactés par l'intermédiaire d'un questionnaire. Ce choix repose sur des justifications empiriques fortes. Nous soutenons en effet l'hypothèse selon laquelle les prix pratiqués par les médecins sont en mesure de dévoiler leurs représentations et leurs comportements. Si l'impact des incitations financières sur le comportement des médecins a abondamment été vérifié (Albouy V. et Déprez M., 2009 ; Janus K. et al., 2008), les études indiquant une relation causale inverse, c'est-à-dire l'adaptation du tarif aux représentations et au comportement des praticiens, sont rares mais pas inexistantes (Batifoulier P. et Gadreau M., 2006b; Grimaldi A. et Vallancien G. 2010). Sur la base de cette hypothèse, l'objectif est de s'appuyer sur les dépassements d'honoraires ordinaires, ceux qui expriment le mieux la conception professionnelle de la pratique tarifaire. La population des omnipraticiens qui, à l'inverse des spécialistes, pratiquent des dépassements assez fréquents mais d'un montant limité (Aballéa P. et al., 2007) semble bien adaptée à cet objectif.

Le questionnaire envoyé (cf. annexe) est de facture classique, il respecte les principes méthodologiques de l'enquête. Le respect des règles du questionnement sociologique a présidé avant tout à l'élaboration du questionnaire : déroulement des questions, distinction des questions de faits et d'opinions, attention portée aux formulations. Le travail s'est en partie inspiré de la grande enquête sociologique quantitative de référence concernant les médecins libéraux (Herzlich C. et al., 1993).

Le questionnaire alterne des questions fermées sur le praticien (sexe, âge, nombre d'enfants à charge...), sur l'environnement du cabinet (zone d'exercice, caractéristique de la patientèle...) sur le mode d'exercice (secteur...), des questions ouvertes (sur leurs opinions quant à la réforme du médecin référent, du médecin traitant, la politique de santé en général...) et des questions mixtes sur leurs pratiques tarifaires (fréquence et motifs de dépassements, d'actes gratuits...). Ces questions ouvertes ont pour objectif de favoriser l'expression des médecins sur leurs pratiques tarifaires et in fine d'y appliquer une analyse textuelle. La succession au sein du questionnaire de questions fermées et ouvertes a paru être une solution intéressante pour appréhender la façon qu'ont les praticiens d'interpréter les règles tarifaires.

Élaboré au printemps 2006, le questionnaire a été envoyé à l'automne de cette même année à 4076 praticiens (2 800 en Île-de-France et 1276 en Bourgogne). La stratégie qui a primé dans la constitution de la population mère a consisté en un échantillonnage aléatoire des omnipraticiens de ces deux régions. Celles-ci sont caractérisées par une sociologie et une démographie médicales différentes et des pratiques tarifaires variées. Elles ont en outre déjà fait l'objet d'études sociologiques (Bloy G., 2005 ; Bloy G., 2004) et économiques (Béjean S., Peyron C. et Urbinelli R., 2002).

Ce travail a bénéficié d'un très bon taux de retour (596 répondants, soit 14,6\%). Ce résultat est supérieur à la norme jugée acceptable pour des études de ce type (Bachimont J., Cogneau J. et Letourmy A., 2006). Il est d'autant plus intéressant que le questionnaire est long (14 pages, 67 questions dont plusieurs ouvertes et certaines délicates - opinions politiques, revenus -) et n'a bénéficié d'aucune incitation (ni rémunération, ni relance). Nous disposons donc d'un matériau riche quantitativement (qui permet un traitement statistique) et qualitativement (les réponses des médecins sont souvent détaillées et passionnées, ce qui permet un traitement lexicométrique et textuel). 
La question de la taille de l'échantillon est également importante. Deux enquêtes antérieures nous permettent de comparer les résultats, quand ils sont comparables. La première a été réalisée par la DREES entre juin et décembre 2002 auprès de 922 médecins généralistes. Les informations recueillies à l'époque portaient sur le patient, le médecin et la consultation (Breuil-Genier P. et Goffette C., 2006; Labarthe G., 2004). La seconde est plus récente (janvier-mars 2006) et porte sur un échantillon de 528 médecins issus du panel Provence-Alpes-Côte d'Azur. Les informations recueillies portent sur la santé publique, la prévention et les opinions quant aux réformes de l'assurance-maladie (Aulagnier et al., 2007; Ventelou B et al., 2005; Videau Y. et al., 2007).

Pour ce travail, nous avons sélectionné 48 questions, dont 25 ouvertes, significatives du discours et des comportements des praticiens face au développement de la marchandisation. Les questions fermées ont subi un premier traitement statistique. Cette première étape nous a permis de dégager les grandes caractéristiques de l'échantillon : secteur, environnement socioprofessionnel, caractéristiques générales de l'activité (nombre de visites et de consultation, durée de la consultation). Nous avons ensuite étudié les pratiques tarifaires des médecins de l'échantillon (dépassements et actes gratuits).

Les questions ouvertes ont, quant à elles, fait l'objet d'une double étude lexicométrique par Sphinx lexica et Alceste. Le premier logiciel est assez général, il offre des fonctions d'analyse textuelle et permet une étude de contenu du corpus. Le logiciel quantifie la fréquence des modalités et des thèmes révélés par l'analyse de contenu. L'analyse lexicométrique permet de mesurer les propriétés sémantiques des réponses, notamment en étudiant la proportion des mots provenant de chaque dimension (intensité lexicale). Enfin, le logiciel offre des possibilités d'analyse factorielle des correspondances multiples permettant de produire des cartes d'association lexicale.

Le second logiciel, Alceste, a permis de compléter et d'affiner l'étude. Celui-ci a pour fonction d'évaluer l'hétérogénéité des phases à partir d'une classification hiérarchique descendante. Ce logiciel propose un traitement différent de la simple analyse de contenu et propose de faire apparaître des phrases (unités de contexte élémentaires- u.c.e.) avec un degré de dissimilitude minimal. Il y a proximité quand deux u.c. partagent un nombre minimal d'occurrences. L'objectif initial est d'étudier les co-occurrences des mots pleins (Reinert M., 2007). L'intérêt principal de cet outil est de considérer le discours comme un acte et c'est pour cette raison que la mise en évidence par le logiciel Alceste des mondes lexicaux est une étape dans l'exploration des mondes sociaux (Didry C., 1998).

\subsubsection{Les caractéristiques de l'échantillon}

L'effectif d'omnipraticiens étudié est composé d'une majorité d'hommes (70\%). Cette répartition est légèrement différente que celle vérifiée au niveau national qui était de $36 \%$ de femmes en 2000 (Darriné S. et Niel X., 2001) et de $39 \%$ en 2008 (Attal-Joubert K. et Vanderschelden M., 2009). Les femmes sont donc sous-représentées. L'effectif est assez âgé puisque $45 \%$ ont plus de 50 ans. La classe modale est celle des $51-55$ ans $(29,1 \%)$ et $42.9 \%$ de l'effectif a une ancienneté dans la profession variant de 16 à 25 ans. Le vieillissement de la profession se vérifie au niveau national puisque la proportion de médecins âgés de 50 à 59 ans est de l'ordre de $40 \%$ en 2006 (Attal-Joubert K. et Vanderschelden M., 2009). Dans son rapport de 2005, l'Observatoire National de la Démographie des Professions Médicales s'inquiétait de la situation de la médecine générale en raison de sa faible attractivité (ONDPS, 2006). 
Les praticiens de l'effectif étudié appartiennent majoritairement, pour 80,5\%, au secteur conventionné (secteur 1) contre 19,5\% au secteur à honoraires différents (secteur 2). Cette répartition est légèrement différente de celle analysée au niveau national qui est de $88 \%$ pour les omnipraticiens et de $92 \%$ pour les généralistes stricto sensu (c'est-à-dire hors médecins à mode d'exercice particulier - MEP' ${ }^{5}$ ). En raison de l'interdiction d'installation en secteur 2 pour les médecins généralistes depuis 1990, le secteur 1 connaît une forte progression en médecine générale (Bories-Maskulova V., 2008). Les praticiens de l'échantillon sont distingués en trois groupes : les médecins en secteur 2, les médecins ayant eu la possibilité de passer en secteur 2 et ayant décidé de rester en secteur 1 (dits secteur 1 choisi, noté 1C) et ceux n'ayant pas eu la possibilité de passer en secteur 2 (dits secteur 1 forcé, noté $1 \mathrm{~F}$ ). Dans l'échantillon étudié, 50,1\% des praticiens exerçant en secteur 1 ont eu la possibilité de passer en secteur 2 parmi lesquels 19,1\% l'ont fait et $31 \%$ ne l'ont pas fait.

Les praticiens de l'effectif exercent dans des quartiers urbains pour une grande majorité d'entre eux $(71,5 \%)$. Le secteur est significativement discriminé par l'environnement $\left(\chi^{2}=60,48\right)$. Les praticiens du secteur 1 exercent majoritairement dans des zones urbaines intermédiaires (26,3\% d'entre eux), urbaines populaires (25\%) et semi-rurales (20\%). Ils sont significativement sous-représentés en zone urbaine résidentielle $(16,4 \%)$. En revanche, les praticiens du secteur 2 exercent pour près de la moitié $(45,2 \%)$ en secteur urbain résidentiel et pour près d'un tiers en secteur urbain intermédiaire. Ils sont significativement sous-représentés dans les quartiers urbains populaires (10,4\% d'entre eux). Leurs modes d'exercice se partagent à égalité entre exercice individuel et collectif. Au niveau national, $39 \%$ des omnipraticiens exercent en groupe (Audric S., 2004).

La durée de consultation est un indicateur de la propension à tarifer l'effort global du praticien (Batifoulier P. et Ventelou B., 2003). La baisse de la durée de la consultation est considérée comme une réponse de certains praticiens de secteur 1 à l'accroissement des contraintes. Plus ces médecins sont insérés dans une logique économique restrictive (comme le secteur 1 pour une bonne partie de la profession), plus leur temps de consultation diminue (Batifoulier P. et Ventelou B. et Protopescu C., 2008). Dans notre échantillon, la durée modale de consultation est représentée par la classe allant de 15 à 20 minutes et représente $44.6 \%$ de l'effectif. La moyenne sur l'ensemble de l'effectif est de 19,6 minutes, ce qui est plus élevé que la moyenne nationale qui est évaluée à 15,6 minutes (Breuil-Genier P. et Goffette C., 2006). Cette particularité s'explique notamment par la surreprésentation du secteur 2 dans l'effectif. La durée de consultation varie positivement avec l'appartenance au secteur. Les médecins de secteur 1 ont une durée de consultation de 18 minutes. Les praticiens exerçant dans le secteur à honoraires libres ont une durée de consultation de 26 minutes en moyenne (contre 18,3 au niveau national). Pour le secteur 1, la moitié de l'effectif a une durée moyenne de 16 minutes. En revanche, pour le secteur 2, plus d'un tiers $(34,2 \%)$ de l'effectif a une durée moyenne de 38 minutes, un autre tiers a une durée de consultation de 21 minutes.

\footnotetext{
${ }^{5}$ Un médecin à mode d'exercice particulier a une spécialité non-reconnue par la Sécurité sociale (acuponcture, homéopathie, ...) ou exerce plusieurs spécialités pour lesquelles il a été qualifié. Il faut donc distinguer les omnipraticiens libéraux (généralistes) et ceux qui pratiquent une médecine plus spécifique. Six praticiens de l'effectif se déclarent spontanément MEP.
} 


\section{Les comportements tarifaires des médecins}

Désireuse de cerner les canaux de transmission de la marchandisation, l'étude s'est plus particulièrement concentrée sur les questions portant sur les différents comportements tarifaires des omnipraticiens. Une première étape a consisté à déterminer les variables susceptibles de discriminer ces comportements. Si la distinction par secteur tarifaire est intuitive, ce critère ne suffit pas (2.1). La seconde étape s'est attachée à l'analyse textuelle des questions ouvertes, en distinguant les actes gratuits des dépassements d'honoraires avant de faire la synthèse des différents comportements types (2.2).

\subsection{Les secteurs tarifaires comme critère de discrimination des comportements}

En introduisant le secteur à honoraires différents, la convention médicale de 1980 a initié une période particulière de dix années au cours desquelles, au-delà du secteur, les praticiens ont dû opter pour des modes d'exercice et des comportements tarifaires différents. L'instauration du secteur 2 (à honoraires différents) ayant eu pour objectif de laisser s'établir un prix de marché (Pierru F., 2007), elle devait donc isoler les praticiens se reconnaissant dans le discours marchand véhiculé dans les politiques de santé. Le secteur tarifaire constitue donc de facto une variable de discrimination du comportement des praticiens. Le passage au secteur 2 est devenu impossible, sauf exception, depuis la convention de 1990, bien que les praticiens du secteur 2 puissent toujours librement revenir en secteur conventionné. Ce gel du secteur 2 et l'asymétrie qu'il génère multiplient alors les comportements tarifaires possibles. Dans ce cas, la distinction par le secteur tarifaire n'est plus opérante puisqu'au sein du secteur conventionné elle ne distingue pas les praticiens ayant librement choisi ce secteur de ceux qui l'auraient choisi ou, au contraire, qui l'auraient quitté pour le secteur 2 s'ils en avaient eu la possibilité.

Les trois questions ouvertes portant sur les motivations des praticiens à exercer leur activité6 ont cependant permis de retrouver statistiquement, par classification ascendante hiérarchique sur données textuelles, les trois classes correspondant aux trois «secteurs » retenus par l'étude, i.e. le secteur 2 (19,5\% de l'effectif total), le secteur 1C $(31 \%)$ et le secteur $1 \mathrm{~F}(47 \%)$. La classification a par ailleurs permis de valider une décomposition du secteur $1 \mathrm{C}$ en praticiens « heureux », satisfaits de leur situation $(29,9 \%)$, et praticiens «mécontents» de cette situation $(17,1 \%)$.

\subsubsection{Le secteur : un critère pertinent ...}

Quel que soit leur secteur tarifaire, les praticiens se disent dans l'ensemble satisfaits à la fois de leur situation et de leurs choix passés. Cette satisfaction apparaît clairement pour ceux qui ont eu la possibilité de choisir leur secteur d'activité. Les médecins du

\footnotetext{
${ }^{6}$ Les trois questions sont les suivantes : (i) Si vous avez eu le choix de votre secteur tarifaire, vous souvenezvous de vos motivations de l'époque? (ii) Qu'est-ce qui aujourd'hui vous satisfait le plus dans votre exercice de la médecine générale? (iii) Qu'est-ce qui vous frustre ou vous inquiète le plus dans cet exercice?
} 
secteur 2 ont ainsi opté sans hésitation $(\mathrm{s}=2,09)^{7}$ pour ce secteur afin de pouvoir exercer librement $(\mathrm{s}=3,53)$ une médecine de qualité en adaptant, sans contrainte financière, la durée de chaque consultation aux besoins des patients. Les praticiens du secteur $1 \mathrm{C}$ sont mus quant à eux par des motivations politiques $(\mathrm{s}=\infty)$ et le refus $(\mathrm{s}=\infty)$ de voir se développer une médecine de «riches » $(\mathrm{s}=\infty)$.

Les médecins du secteur $1 \mathrm{~F}$ motivent quant à eux leur satisfaction à exercer dans ce secteur par la simplicité de la tarification. Bien qu'insuffisants $(\mathrm{s}=2,41)$, imposés $(\mathrm{s}=2,01)$ à un niveau très bas $(\mathrm{s}=1,87)$ et peu évolutif $(\mathrm{s}=2,25)$, les honoraires conventionnés qu'ils pratiquent sont à la base de l'égalité des soins $(\mathrm{s}=2,11)$ et leur permettent de ne pas se poser de question $(\mathrm{s}=\infty)$ sur le montant $(\mathrm{s}=2,25)$ qu'ils doivent faire payer $(\mathrm{s}=1,93)$.

Le même argumentaire émerge dans l'analyse des motifs de satisfaction et de mécontentement. Ainsi, si la majorité des médecins avance la relation avec le patient (cité par 45,3\% des praticiens) comme premier motif de satisfaction, la notion de liberté apparaît en seconde position (19,6\%). De même, c'est la charge administrative $(30,7 \%)$, les difficultés rencontrées dans les relations avec les autres acteurs $(19,4 \%)$ et le manque de temps $(12,7 \%)$ qui gênent le plus.

Plus précisément, les médecins du secteur 2 sont satisfaits de leur mode d'exercice car il leur permet de réaliser une médecine utile $(\mathrm{s}=1,81)$ avec une bonne relation médecinpatients $(\mathrm{s}=2,44)$ et en répondant à la demande $(\mathrm{s}=2,03)$ des patients (écoute $(\mathrm{s}=2,32)$, temps $(\mathrm{s}=1,69)$, horaire $(\mathrm{s}=1,74)$, technicité $(\mathrm{s}=2,44))$. Cette efficacité est attestée par une certaine reconnaissance $(\mathrm{s}=1,63)$ et de la satisfaction $(\mathrm{s}=2,03)$. Ces praticiens sentent toutefois leurs libertés rognées par la bureaucratie $(\mathrm{s}=2,78)$, une charge de travail $(\mathrm{s}=1,94)$ importante et souffrent d'une mauvaise reconnaissance de la valeur $(\mathrm{s}=2,70)$ de leur travail.

Les médecins du secteur 1C insistent pour leur part sur le plaisir $(\mathrm{s}=1,79)$ qu'ils éprouvent à fournir des soins qu'ils jugent de qualité ( $\mathrm{s}=1.68)$ grâce à l'élaboration d'un bon diagnostic $(\mathrm{s}=2,39)$ leur permettant d'accompagner $(\mathrm{s}=1,99)$ les patients et de leur proposer une bonne prise en charge $(\mathrm{s}=1,49)$. Mais, ces praticiens ressentent un malaise se traduisant par l'impression ( $\mathrm{s}=2,26)$ d'être un simple rouage mal considéré $(\mathrm{s}=1,80)$ par les autres acteurs et se traduisant par une augmentation des risques $(\mathrm{s}=2,02)$ sanitaires et juridiques. Le malaise découle d'abord du comportement des pouvoirs publics $(\mathrm{s}=2,02)$, qui multiplient les contrôles ( $\mathrm{s}=1,72)$, organisent la concurrence ville-hôpital $(\mathrm{s}=2,02)$. Ce malaise découle aussi de l'opposition exacerbée entre généralistes et spécialistes, du niveau d'exigence $(\mathrm{s}=2,35)$ croissante des patients et de la politique du médicament $(s=2,19)$ qui pèse sur la prescription.

C'est en premier lieu la richesse $(\mathrm{s}=\infty)$ des contacts humains $(\mathrm{s}=1,65)$ qui est mise en avant par les praticiens du secteur $1 \mathrm{~F}$. Cet enrichissement repose sur la conjonction de la diversité $(\mathrm{s}=1,46)$ clinique $(\mathrm{s}=2,04)$ et la diversité du champ d'action $(\mathrm{s}=1,86)$ d'une part, la pratique libérale $(\mathrm{s}=1,43)$, alliant autonomie $(\mathrm{s}=1,91)$, liberté d'organisation $(\mathrm{s}=1,52)$ et liberté de gestion $(\mathrm{s}=1,49)$ d'autre part. Bien que les effets soient moins marqués, les prati-

\footnotetext{
${ }^{7}$ Les mots ou groupes de mots les plus spécifiques des réponses de chaque catégorie de médecins et ayant un $\chi^{2}$ significatif à $1 \%$ sont indiqués en italique. L'indice $s$ de spécificité (entre parenthèses), fourni par Sphinx, est le rapport entre le nombre d'observations d'un mot et le nombre théorique d'utilisations tel qu'il résulterait d'un emploi proportionnel au nombre total de mots prononcés par la catégorie. L'indice est supérieur à 1 lorsqu'il est sur-utilisé par la catégorie, inférieur à 1 lorsqu'il est sous-utilisé surutilisé et infini lorsqu'il est utilisé par cette seule catégorie.
} 
ciens n'ayant pas pu choisir leur secteur sont cependant sensibles aux diverses contraintes déjà évoquées par les autres praticiens. Ils font face à une surcharge de travail (manque de temps $(\mathrm{s}=1,36)$, souffrent d'une dévalorisation $(\mathrm{s}=1,62)$ de leur activité et sont confrontés à des difficultés dans leurs relations avec l'assurance maladie $(\mathrm{s}=1,38)$ Leur principal regret concerne cependant l'émergence d'un consommateur $(\mathrm{s}=\infty)$ de soins dont les exigences $(\mathrm{s}=1,55)$ sont en constante augmentation $(\mathrm{s}=1,40)$.

\subsection{2. ... mais ne tenant pas compte de toutes les lignes de clivage}

Les discours dominants de chaque catégorie de médecins sont résumés sur le Graphique 1. Celui-ci, issu d'une AFC globale des variables portant sur les motivations liées à l'exercice de la médecine, met en évidence un double clivage. Le premier, visible le long du premier axe du graphique, oppose les praticiens ayant eu la possibilité de choisir leur secteur d'activité des autres. Il montre la richesse de la réflexion et du questionnement qui a nourri le choix du secteur tarifaire et l'oppose à l'absence de jugement des « jeunes » médecins, auxquels la question ne se pose pas. L'importance statistique de cet axe (qui explique 54,3\% de l'inertie du nuage de mots) comme le vocabulaire déployé, notamment par ceux qui ont choisi de rester en secteur 1, souligne le rôle historique de la parenthèse 1980-1990 dans la représentation que les médecins se font de leur profession.

Le second clivage, représenté par l'axe vertical du graphique, oppose la dimension individuelle de l'activité des médecins à la dimension collective de cette activité. Le côté individuel s'appuie sur l'idée selon laquelle la qualité de la médecine repose sur l'expertise et le savoir-faire de la profession. Propriétés qu'il faut donc préserver, dans l'intérêt des patients. Le côté collectif envisage la qualité sous un angle plus social, voire politique. Celle-ci émerge à la fois d'une organisation efficace et juste des soins. À côté de sa situation médicale, la situation sociale, familiale ou financière du patient prend alors une importance inexistante dans la dimension individuelle. Ce second clivage regroupe les secteurs $1 \mathrm{C}$ et $1 \mathrm{FH}$ (secteur 1F, composé de praticiens heureux) du côté de la dimension collective de l'activité et les secteurs 2 et 1FM (secteur 1F, composé de médecins mécontents) du côté de la dimension individuelle.

Les motivations ainsi révélées, parce qu'elles conduisent les acteurs à mobiliser les justifications qui sous-tendent leurs choix, peuvent être rapprochées des catégories de discours mobilisés dans les conventions médicales (Abecassis P. et Domin J.-P., 2008; Abecassis P. et Domin J.-P., 2009). Les praticiens du secteur 2 privilégient le discours libéral traditionnel, marqué par les principes de liberté d'exercice, de liberté de tarifs et de liberté d'organisation. Sur le Graphique 1, ces praticiens se positionnent naturellement dans le cadran inférieur gauche, à l'intersection de la dimension individuelle et du choix volontaire. Ce positionnement illustre alors clairement l'idée selon laquelle la pratique libérale est garante d'un bon niveau de soins.

Les médecins du secteur $1 \mathrm{C}$, ayant choisi volontairement de rester en secteur à honoraires conventionnés, se positionnent eux aussi dans la partie gauche du graphique, c'est-à-dire du côté du choix. Mais ils considèrent que leur profession ne peut se cantonner au seul savoirfaire technique, leur conception de l'éthique professionnelle les conduit à tenir compte des aspects sociaux et politiques. On reconnaît ici les ingrédients du discours libéral renouvelé. 
Les médecins des secteurs $1 \mathrm{~F}$ n'ayant pas eu la possibilité de choisir leur secteur tarifaire sont regroupés sur la droite du graphique. La population est cependant clairement discriminée par l'axe 2 distinguant les praticiens qui, si on leur avait donné le choix, auraient opté pour le secteur 2 de ceux qui auraient choisi de rester en secteur conventionné. La position des premiers les rapproche du secteur 2 et du discours libéral traditionnel, mais le discours est contraint par les obligations liées au secteur 1. De la même façon, les praticiens du secteur $1 \mathrm{FH}$ ont une posture proche de ceux du secteur $1 \mathrm{C}$ et du discours libéral renouvelé. Toutefois, ces deux catégories sont associées à un vocabulaire peu étoffé, limité à de simples jugements (aimer ou non, convenir ou non) sans justification.

\section{Graphique 1 - AFC sur données textuelles : Motivations du choix du secteur en fonction du secteur choisi}

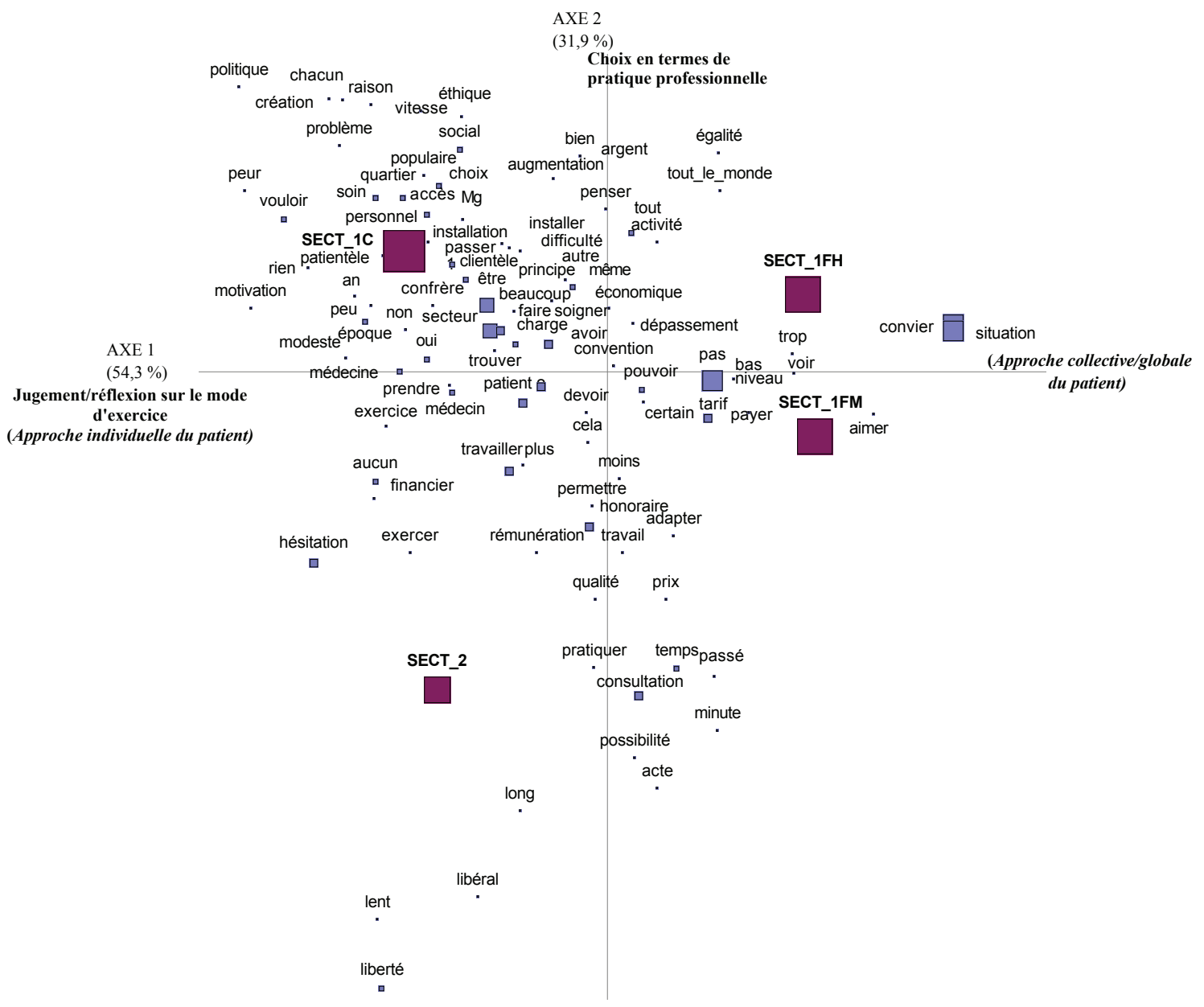




\subsection{Actes gratuits et comportements tarifaires : deux aspects du comportement marchand des médecins.}

Le discours marchand n'est spécifique d'aucune des catégories de médecins. Ce résultat ne surprend pas, la référence au marché n'ayant jamais été absente des revendications des praticiens. De même, aucune catégorie de médecins ne semble rejeter catégoriquement les politiques de santé. Celles-ci fournissent en effet des leviers d'action permettant aux praticiens d'harmoniser leurs pratiques à leurs discours. Le degré d'adoption des deux leviers que sont les actes gratuits et les dépassements d'honoraires peut alors être interprété comme un indicateur d'appropriation ou de rejet l'évolution marchande des politiques de santé.

\subsubsection{Les actes gratuits: des actes courants aux motifs multiples}

Les omnipraticiens déclarent majoritairement $(89,8 \%)$ réaliser des actes gratuits, mais cette pratique est plus fréquente dans le secteur 2. Pour tous les secteurs, un tiers des praticiens effectue moins de 5 actes gratuits par mois, les autres se répartissent de façon assez homogène sur un intervalle allant de 5 à 25 actes gratuits par mois. Parmi les individus qui ne font pas d'actes gratuits, seuls 10,9\% sont issus du secteur 2 (alors qu'ils représentent 19,6\% de notre échantillon) et $89,1 \%$ sont issus du secteur 1 (alors qu'ils représentent $80,4 \%$ de l'échantillon). Parmi les praticiens qui ne font pas d'actes gratuits, $59 \%$ en ont déjà fait contre $41 \%$ qui n'en ont jamais fait dans le passé, ceci indépendamment du secteur.

L'évolution de la pratique des actes gratuits est un indicateur significatif de la marchandisation. Celle-ci mènerait les médecins à tarifer des actes qu'ils réalisaient gratuitement auparavant (Batifoulier P. et Ventelou B., 2003). Pour la majorité des praticiens de notre échantillon, $(81,1 \%)$ il n'y a pas de changement. En revanche, $14,8 \%$ des praticiens ont bien le sentiment d'une réduction du nombre d'actes gratuits. Mais la dépendance au secteur est significative. Pour le secteur 1 dans son ensemble, aucun médecin n'a l'impression d'en faire plus, mais 14,2 \% ont l'impression d'en faire moins. Le secteur 2 est le seul secteur dans lequel certains praticiens $(18,7 \%$ des médecins du secteur 2 , soit $4,1 \%$ du total de l'échantillon) disent faire plus d'actes gratuits qu'auparavant.

$\mathrm{Si}$ au sein du secteur 1 , tous les praticiens font très majoritairement des actes gratuits ( $88 \%$ des praticiens du secteur 1C, $92,3 \%$ de ceux du secteur $1 \mathrm{FH}$ et $94,1 \%$ de ceux du secteur $1 \mathrm{FM}$ ), les différences sont plus marquées parmi ceux qui n'en font pas. En effet, alors que tous les généralistes du secteur 1FM qui ne pratiquent pas d'actes gratuits en réalisaient dans le passé, ils ne sont que plus que 76,6\% dans le secteur $1 \mathrm{C}$ et $56 \%$ dans le secteur $1 \mathrm{FH}$ à être dans ce cas. Une distinction de même nature entre secteurs se retrouve dans l'évolution du nombre d'actes gratuits des praticiens déclarant continuer à en réaliser. (Graphique 2).

Au total, deux groupes se distinguent, les généralistes du secteur 2 qui sont les seuls pour qui le nombre d'actes gratuits effectués augmente et les praticiens du secteur 1FM qui ont une position diamétralement opposée puisque $27,9 \%$ d'entre eux ont soit réduit soit totalement arrêté les actes gratuits. Les autres secteurs se rapprochent du secteur 1FM mais de façon moins prononcée puisque le taux d'individus ayant réduit ou supprimé les actes gratuits y est inférieur (respectivement $18 \%$ et $17 \%$ pour les secteurs $1 \mathrm{C}$ et $1 \mathrm{FH}$ ). 
La compréhension des comportements qui sous-tendent ces clivages peut être en partie fournie par l'analyse de ce qui motive la pratique et l'évolution des actes gratuits. Celle-ci montre une certaine homogénéité des discours et des comportements quelle que soit la catégorie de médecins ${ }^{8}$ et l'on peut faire émerger des classes d'arguments avancés de façon plus ou moins prononcée par tous les praticiens. Les actes gratuits sont en effet motivés pas quatre types d'arguments. Les premiers, les plus fréquents, représentent $33 \%$ des unités de contexte élémentaires (u.c.e.) retenues par la classification ascendante hiérarchique réalisée par Alceste, les motivations des actes gratuits regroupés dans cette classe sont de nature purement technique. On y trouve des arguments tels que la vaccination (citée 75 fois), la lecture de résultats de laboratoires ou d'imagerie médicale (citée 117 fois).

La seconde classe (représentant $30 \%$ des u.c.e.) est formée des motivations liées à la situation financière du patient. Y sont évoqués les dépannages financiers et les difficultés financières des patients (citées 102 fois), la CMU (cités 27 fois), les difficultés sociales (citées 14 fois).

\section{Graphique 2 - Evolution déclarée du nombre d'actes gratuits en fonction du secteur}

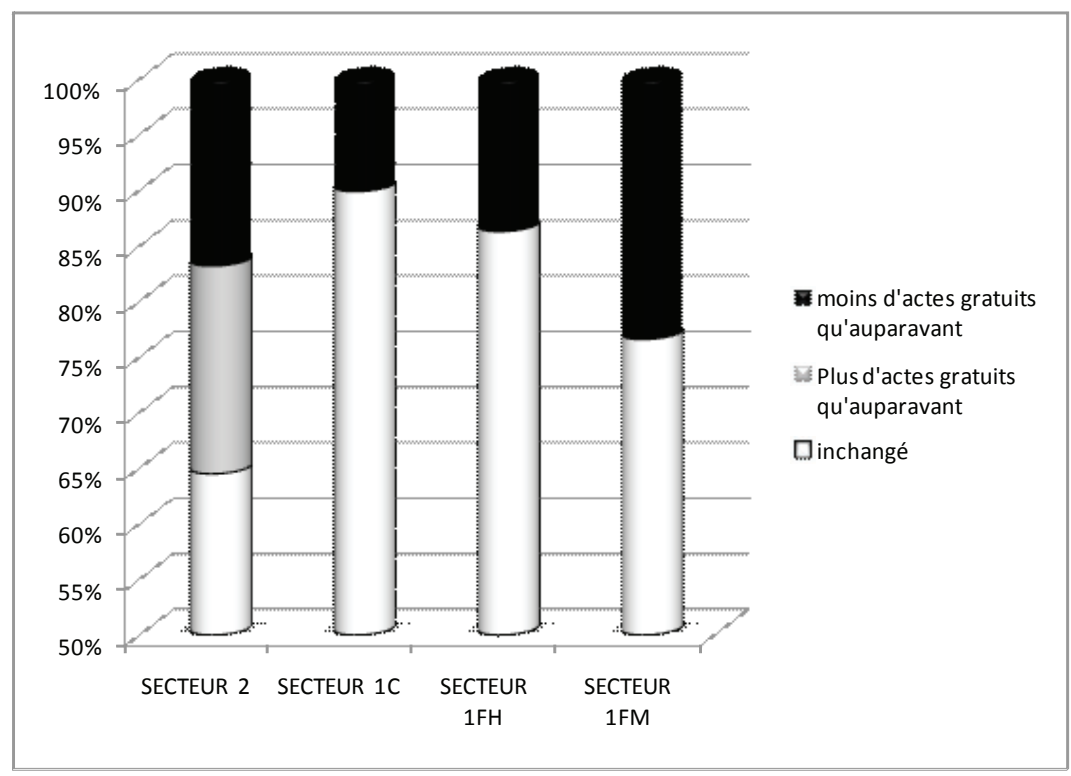

La troisième classe (20 \% des u.c.e.) rassemble les raisons de nature commerciale et marchande. Sont ainsi évoqués le suivi de consultations (cité 19 fois) ou les renouvellements d'ordonnance (cités 84 fois). Mais d'autres raisons sont évoquées dans cette classe, des actes administratifs (certificats, cités 158 fois, lettres aux confrères, citées 60 fois), des actes par téléphone (cités 20 fois) ou des actes jugés courts, simples ou rapides (cités 91 fois). Ce qui caractérise les arguments de cette classe, c'est que les médecins ne sont pas en mesure de

\footnotetext{
${ }^{8}$ Les tests de dépendance du $x^{2}$ des questions portant sur les actes gratuits au type de praticiens ne sont significatifs qu'à $10 \%$.
} 
tarifer les actes. Soit ce ne sont pas des actes médicaux, les praticiens ne disposent pas des capacités ou des outils d'évaluation adéquats. Soit il y a impossibilité physique de perception de l'honoraire, comme dans le cas de la «consultation» téléphonique. Soit enfin la valeur estimée par le praticien est inférieure à celle d'une consultation au tarif conventionné ${ }^{9}$ pour lequel le médecin ne peut, techniquement percevoir de juste rémunération

La quatrième classe (16\% des u.c.e.) regroupe les motivations liées à une spécificité non médicale du patient, comme lorsque plusieurs membres de la même famille viennent consulter en même temps (cité 103 fois) et lorsque le patient est un proche, un ami (cités 23 fois) ou un confrère (cité 9 fois).

Comme l'illustre le tableau ci-dessous, les médecins du secteur 2 se caractérisent par l'absence de motivations liées à une propriété particulière du patient et une répartition homogène des arguments dans les trois autres classes. Les praticiens du secteur $1 \mathrm{C}$ ont un profil proche de celui des précédents, mais leurs motivations embrassent un spectre plus large alors que les praticiens du secteur $1 \mathrm{~F}^{10}$ motivent plus volontiers leurs actes gratuits par des arguments liés à une spécificité non médicale des patients.

Tableau 1- Motivations des actes gratuits

\begin{tabular}{|c|c|c|c|}
\hline $\begin{array}{ll}\text { Classes de motivations } & \text { Catégorie de praticiens } \\
\end{array}$ & Secteur 2 & Secteur 1C & Secteur 1F \\
\hline Classe 1 : Motivations techniques & $+++(2,64) *$ & $+++(2,42)$ & $++(1,56)$ \\
\hline $\begin{array}{l}\text { Classe } 2 \text { : Motivations liées à la situation financière } \\
\text { des patients }\end{array}$ & $+++(2,64)$ & $+++(2,32)$ & $++(1,87)$ \\
\hline Classe 3 : Motivations commerciales /marchandes & $+++(2,09)$ & $++(2,42)$ & $+(1,95)$ \\
\hline $\begin{array}{l}\text { Classe } 4 \text { : Motivations liées à une propriété } \\
\text { spécifique non médicale du patient }\end{array}$ & & $+(1,45)$ & $+++(1,95)$ \\
\hline
\end{tabular}

Pour être correctement expliquées, les différences ainsi observées doivent être corrélées aux caractéristiques de la clientèle d'une part et au lieu d'exercice d'autre part. Ces deux variables sont en effet susceptibles de créer des distorsions dans les résultats. Ainsi, le fait que les médecins du secteur 2 soient insensibles aux propriétés spécifiques des patients (classe 4) pourrait s'expliquer par un comportement éthique consistant à ne pas discriminer ses patients. Un tel comportement peut aussi découler d'une sur représentation de certaines

\footnotetext{
9 Il est à noter que les classes ainsi construites ne sont pas totalement exclusives les unes des autres, les actes techniques en particulier peuvent contenir une dimension marchande dans le sens par exemple où plusieurs de ces actes peuvent aussi être courts.

${ }^{10}$ En termes de motivations, il n'apparait pas de différence significative entre les secteurs 1FH et 1FM.
} 
catégories de populations (célibataires ou familles de petite taille) propres à certaines zones d'exercice (urbaine résidentielle) où les médecins du secteur 2 sont majoritairement implantés. À l'inverse, l'implantation en zone rurale ou semi rurale, la patientèle formée de familles, d'amis ou de connaissances ainsi que des comportements fortement discriminatoires seraient en mesure d'expliquer la sur détermination des motivations liées aux spécificités des patients par les médecins du secteur $1 \mathrm{~F}$.

En faisant l'hypothèse que les actes gratuits et les dépassements d'honoraires sont de même nature, l'analyse des dépassements devrait compléter les résultats obtenus sur les actes gratuits et permettre de lever les incertitudes sur l'interprétation des motivations des acteurs.

\subsubsection{Dépassements d'honoraires : I'apanage du secteur 2}

En matière de dépassements d'honoraires, la différence juridique ${ }^{11}$ entre secteur 1 et 2 mène naturellement l'analyse à relever une forte dépendance au secteur d'appartenance du praticien. Ainsi, si, au total $69 \%$ des praticiens de l'effectif appliquent le tarif conventionnel, ils sont $90,4 \%$ des praticiens du secteur 1 à ne pas pratiquer de dépassement. À l'opposé, seuls $5,5 \%$ des praticiens du secteur 2 appliquent effectivement le tarif conventionnel.

Lorsqu'il existe, le dépassement reste modeste dans le secteur 1 où seuls $1,3 \%$ des praticiens de ce secteur dépasse de plus de $25 \%$ le tarif conventionnel. À l'inverse, les dépassements sont donc significativement élevés dans le secteur 2. 86,3\% des médecins de ce secteur dépassent de plus de $25 \%$ le tarif conventionnel, et 50,5\% d'entre eux le dépassent de plus de $50 \%$.

Si on ne s'intéresse qu'au secteur 1, l'environnement du cabinet est significatif. En zone urbaine résidentielle, les dépassements sont peu nombreux (8,2\% des praticiens). On peut considérer qu'il s'agit d'un positionnement concurrentiel. À l'inverse, il n'y a aucun dépassement en zone rurale et, en zone semi-rurale, les dépassements restent marginaux $(2,2 \%)$, Enfin $12,1 \%$ des praticiens de ce secteur pratiquent des dépassements de plus de $10 \%$ dans les zones urbaines populaires. Dans ces zones, la concurrence des praticiens de secteur 2 est inexistante, ces dépassements traduisent donc l'absence de positionnement concurrentiel.

Au sein de ce secteur 1, les médecins de la catégorie 1FH se distinguent des autres. Toutes proportions gardées, ces derniers sont les moins nombreux du secteur 1 à pratiquer des dépassements $(8,1 \%$ d'entre eux) et les dépassements sont particulièrement faibles puisque plus de la moitié des praticiens $(53,8 \%)$ qui dépassent déclarent dépasser de moins de $10 \%$ du tarif conventionnel et $92,3 \%$ dépassent de moins de $25 \%$ du tarif conventionnel.

À l'inverse, les médecins des catégories $1 \mathrm{C}$ et $1 \mathrm{FM}$ sont plus nombreux à pratiquer des dépassements $(10,2 \%$ pour le secteur $1 \mathrm{C}, 11,1 \%$ pour le secteur $1 \mathrm{FM})$ que pour la catégorie 1FH et les dépassements sont plus élevés. La majorité des praticiens déclarant effectuer des dépassements dans ces deux secteurs, soit 77,8\% (secteur 1C) et 63,6\% (secteur 1FM) se situent dans la classe allant de $10 \%$ à moins de $25 \%$ du tarif conventionnel. Cependant, alors qu'en moyenne 19,3\% des praticiens du secteur 1 dans son ensemble déclarent pra-

\footnotetext{
11 Si les médecins exerçant en secteur 2 peuvent pratiquer des honoraires libres, ceux du secteur 1 doivent pratiquer les tarifs conventionnels, sauf exigence particulière du patient. Dans cette étude, nous considérons que tous les honoraires supérieurs aux tarifs conventionnels sont des dépassements d'honoraires, indépendamment du secteur tarifaire.
} 
tiquer plus de dépassements qu'auparavant, ils sont plus du quart $(25,4 \%)$ du secteur $1 \mathrm{C}$ à avoir ce sentiment.

L'analyse ascendante hiérarchique réalisée avec Alceste des questions spécifiques aux dépassements d'honoraires fait émerger quatre classes. La première (20\% des u.c.e.) est la plus riche en vocabulaire, elle regroupe les termes et expressions tarifaires ainsi que les spécificités financières ou personnelles des patients motivant les variations de ces tarifs (critères socio-financiers). On trouve ainsi des modulations de dépassement d'honoraires en fonction de la couverture maladie (CMU, citée 17 fois ; mutuelle, citée 11 fois), en fonction de la situation financière (situation difficile, citée 11 fois; difficulté financière, citée 17 fois, revenu, cité 11 fois) ou en fonction de la situation personnelle (étudiant, cité 5 fois).

La seconde classe (14\% des u.c.e.) rassemble les motivations normatives liées au comportement des patients. Les praticiens attendent certains comportements de leur patientèle et sanctionnent les manquements par des dépassements. On trouve ainsi dans cette classe, les termes tels que motif valable (cité 46 fois), les déplacements injustifiés (cités 17 fois), les visites abusives (citées 32 fois).

La troisième classe (15\% des u.c.e.) regroupe les jugements des médecins sur les dépassements de leurs confrères. Le vocabulaire ne constitue pas directement une motivation aux dépassements, mais donne une information sur les représentations des médecins. La classe comprend ainsi principalement un vocabulaire de jugement positif ( « une bonne chose » ou « une bonne idée »; cités 25 fois, «j' approuve » ou « je comprends », cités 24 fois). Les termes négatifs sont moins nombreux et moins significatifs (éviter, cité 9 fois; abus, cités 9 fois).

La dernière classe est la plus fréquente (51\% des u.c.e.). Elle rassemble principalement les motifs prévus par la convention médicale au dépassement d'honoraires. Les visites sont ainsi très fréquentes, (citées 103 fois), ainsi que les exigences particulières (citées 68 fois) ou les déplacements (cités 17 fois).

Le Graphique 3 fait apparaître la fracture en fonction du secteur $(79,7$ \% de l'inertie du nuage de mots est expliquée par l'axe 1). Les praticiens du secteur 2, jouant à plein la carte de la liberté des tarifs qui leur est offerte, les modulent librement en fonction des critères socio-financiers mis en évidence par la première classe. De leur côté, les médecins du secteur 1 motivent principalement leurs dépassements d'honoraires en fonction du comportement du patient. Toutefois, l'axe 2 , en opposant le vocabulaire des seconde et dernière classes de mots, montre la position singulière des praticiens du secteur 1FM qui se conforment plus fréquemment aux cas de dépassement prévus par la convention médicale. Les praticiens des secteurs $1 \mathrm{C}$ et $1 \mathrm{FH}$ prennent plus de libertés puisqu'ils utilisent les dépassements comme des incitations au civisme des patients.

\subsubsection{Deux effets et deux paradoxes de l'infiltration du marchand dans les comportements tarifaires}

L'analyse globale des motivations a montré l'opposition entre d'une part un groupe formé du secteur 2 et du secteur 1FM, et, d'autre part, les secteurs $1 \mathrm{C}$ et $1 \mathrm{FH}$. Le premier groupe mobilise principalement le discours libéral, même si les médecins du secteur 1FM modifient légèrement leurs motivations pour l'adapter aux contraintes de leur situation. Le second groupe se reconnaît dans le discours libéral renouvelé. 


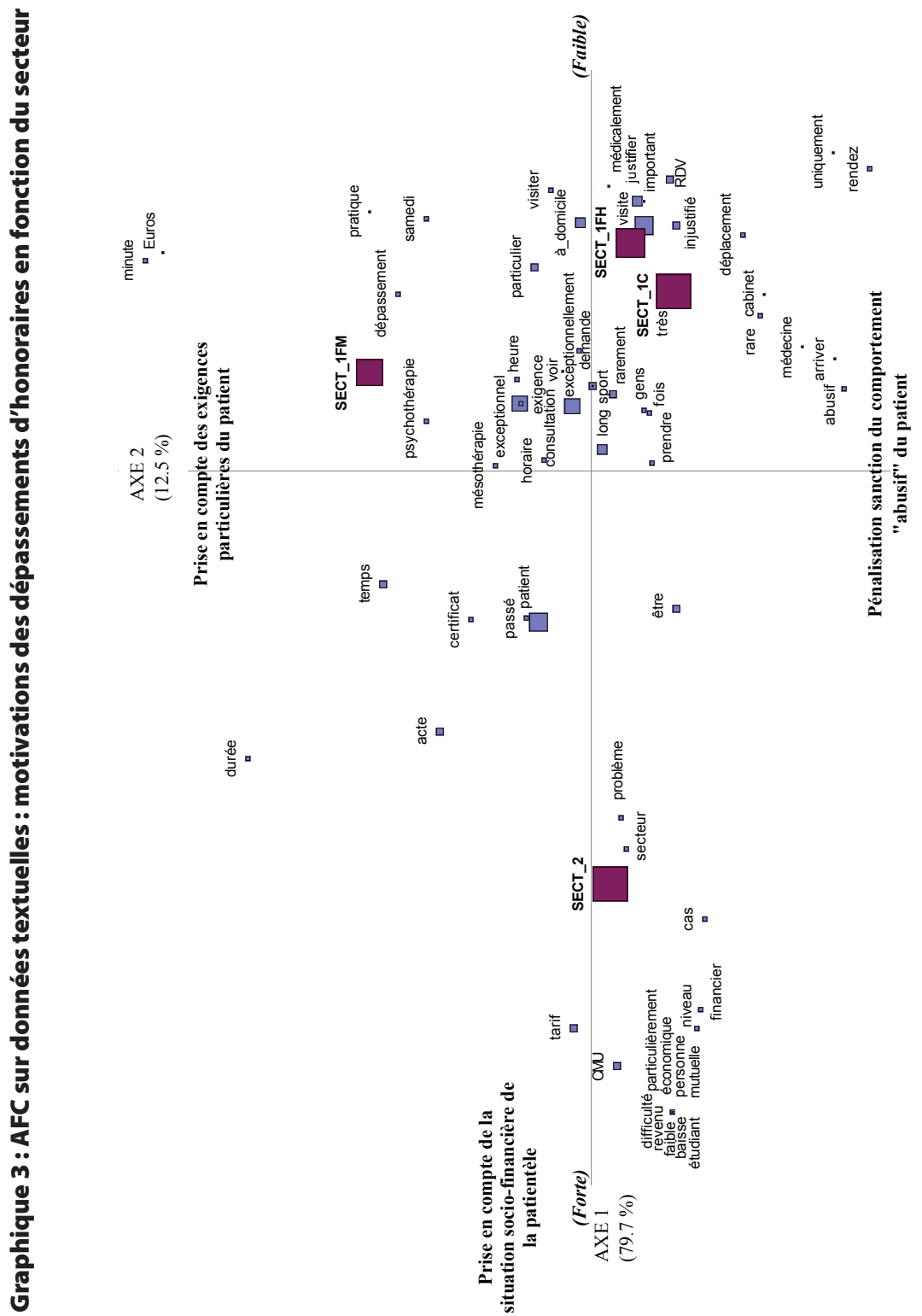

Si cette opposition est flagrante pour ce qui concerne les questions liées aux motivations à l'exercice de la médecine, elle n'apparaît plus aussi clairement lorsque les médecins motivent leurs actions en termes d'actes gratuits et de dépassement d'honoraires. Dans ce cas, le pragmatisme des acteurs lié à leur situation et à leurs représentations module en effet les discours et fait émerger des comportements singuliers, voire contradictoires avec les discours sous-jacents. L'étude des questionnaires a pu mettre en exergue deux effets et deux paradoxes liés aux comportements tarifaires. Ainsi, le discours marchand, en s'infiltrant via les comportements des acteurs, fait bouger les lignes « traditionnelles » de partage entre secteurs d'une part, entre espaces discursifs d'autre part. 


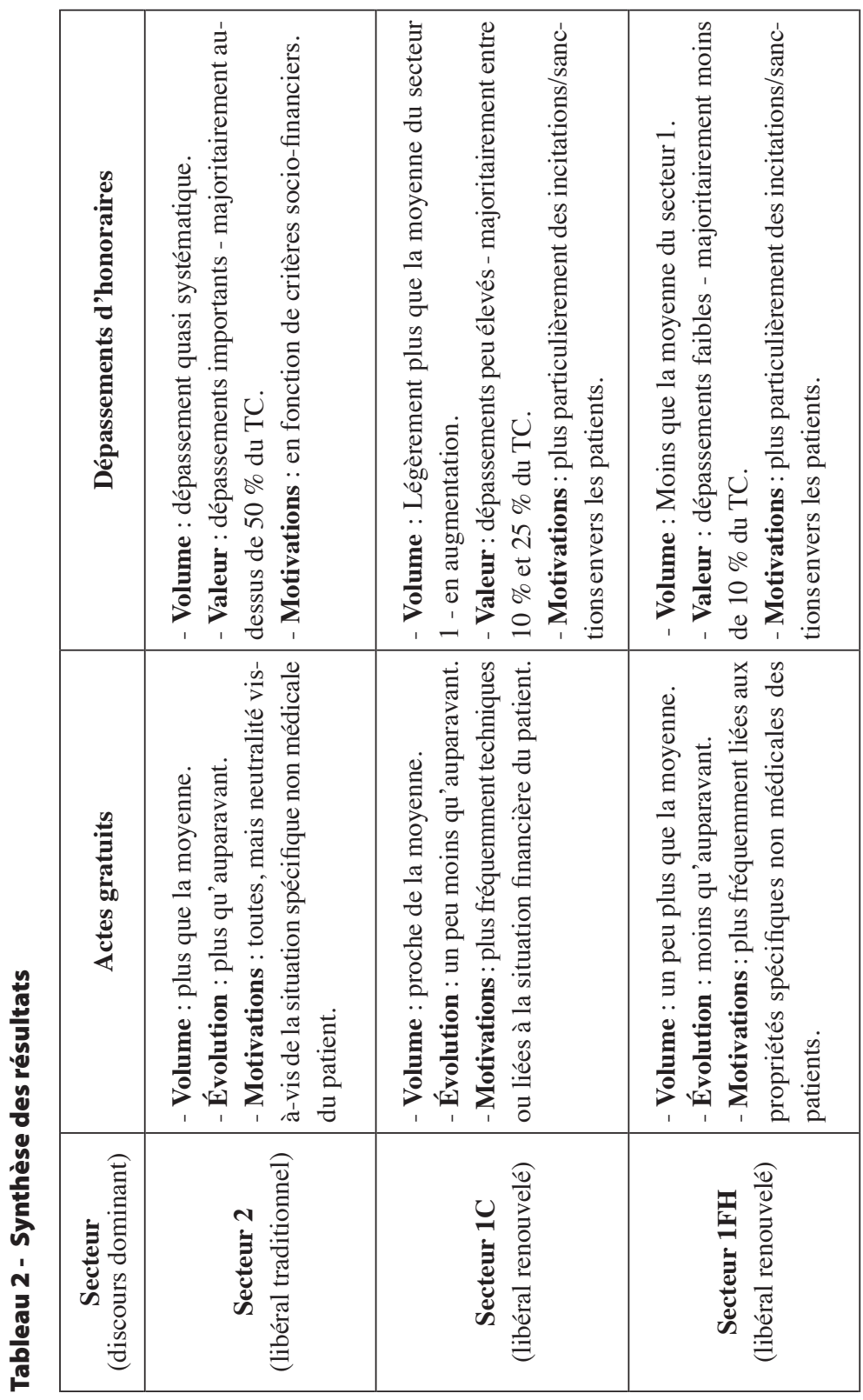

Le premier effet de la marchandisation est dévoilé par la position singulière du secteur 2 qui se distingue des autres secteurs en adoptant un comportement atypique d'augmentation du nombre des actes gratuits tout en maintenant un taux important de dépassement d'honoraires (cf. Tableau 2). La marchandisation prend dans ce cas une forme conforme au discours libéral traditionnel soutenu par ces médecins. En effet, le discours libéral traditionnel laisse au praticien le soin de juger le montant adéquat de ses honoraires, « avec tact et mesure ». Son jugement peut donc mener le praticien à pratiquer exceptionnellement des actes gratuits ou des tarifs d'hono- 
raires particulièrement élevés. Le principe de justice qu'il faut mobiliser pour établir le bon tarif d'honoraire n'est cependant pas édicté par le discours libéral. Aussi, s'il n'est pas surprenant de trouver une référence à la situation financière du patient (le médecin s'engageant, par le serment d'Hippocrate à « soigner l'indigent »), la neutralité des médecins du secteur 2 aux particularités non médicales et non financière du patient est plus inattendue. L'hypothèse de marchandisation fournit une explication plausible. La liberté tarifaire focalise en effet l'attention du médecin sur le tarif et, parce qu'il n'existe pas de marché de la santé, celui-ci ne peut s'imposer naturellement au médecin. Chaque acte médical doit donc faire l'objet d'une évaluation et d'une négociation tarifaire spécifique. Dans ce cadre, seuls les mécanismes de marché sont, a priori, en mesure de satisfaire à la fois les contraintes éthiques («avec tact et mesure ») et les contraintes comptables imposées par les tutelles. Aussi, à l'instar d'une négociation marchande, le prix est calculé selon les règles du marché où, pour que le prix soit «juste», les aspects personnels indépendants de la transaction ne peuvent ni ne doivent interférer.

Le marché et ses mécanismes sont aussi à la base du second effet mis en évidence dans cette analyse. Pour déterminer quels actes doivent être prodigués gratuitement ou quels autres doivent faire l'objet d'un dépassement d'honoraires, le praticien doit être en mesure d'évaluer la valeur de ses prestations. Les dispositifs issus du discours marchand, tels que les suppléments tarifaires inscrits par la convention médicale, permettent aux médecins d'évaluer des actions qu'ils ne savaient évaluer avec précision auparavant ${ }^{12}$. Or, En attribuant une valeur à des actes jusque-là non évalués ou difficilement évaluables, le discours marchand permet aux médecins de tarifer ce qui était gratuit et contribue à réduire le nombre d'actes gratuits.

Cette dynamique va dans le sens du discours libéral, traditionnel comme renouvelé, qui s'oppose, par nature, à la gratuité des soins, à l'exception des situations exceptionnelles, dont le caractère d'exception est laissé au jugement du praticien. En réduisant quantitativement le nombre d'actes gratuits par rapport aux autres et en justifiant ces actes par des motifs principalement liés aux caractéristiques du patient, les médecins du secteur 1FM épousent alors parfaitement ce discours. Si leur jugement est bien mobilisé pour les actes gratuits, ces médecins se contentent, en partie par résignation, des multiples majorations fournies par les conventions médicales pour évaluer leurs tarifs et les dépassements éventuels. C'est à ce titre que cette catégorie de praticiens se dit la moins concernée par les tarifs (dès lors qu'il est jugé suffisant) et effectue les dépassements d'honoraires les moins importants.

La difficulté à évaluer la valeur des actes médicaux nourrit le premier paradoxe de la marchandisation : l'accroissement du nombre d'actes gratuits des praticiens disposant de la liberté des tarifs. Les résultats de notre analyse montrent en effet que ce comportement n'est pas dû à une surreprésentation des catégories de patients les plus démunis dans la clientèle du secteur 2 et, si un effet «bonne conscience » peut être envisagé, il ne peut être étayé par l'analyse des motivations qui excluent précisément les discriminations non justifiées médicalement ou financièrement.

Nous avançons donc l'hypothèse que ces actes gratuits découlent d'une double imperfection du «marché ». La première imperfection est liée à la discontinuité des honoraires. Ceux-ci sont libres au-dessus du tarif conventionnel, mais celui-ci constitue une borne

\footnotetext{
${ }^{12}$ La convention médicale propose par exemple une majoration kilométrique différente en plaine $(0,61 € / \mathrm{km})$ ou en montagne $(0,91 € / \mathrm{km})$ ou une majoration de déplacement de nuit différente selon que le déplacement a lieu de $20 \mathrm{~h}$ à minuit (38€45) ou de minuit à $6 \mathrm{~h}(43 € 50)$.
} 
inférieure infranchissable légalement car elle se heurte à l'impossibilité de demander le remboursement d'une fraction de ce tarif à l'assurance-maladie. La seconde imperfection, déjà évoquée, relève du manque d'information ou de formation des médecins qui ne savent pas évaluer des tâches non médicales (courriers, certificats...) ou ne disposent pas de moyens techniques de facturation de certains autres actes (téléphone, mail,...). Lorsqu'ils sont confrontés à des situations d'imperfection de ce type les médecins renoncent à percevoir des honoraires ou demandent des honoraires minima (correspondant au tarif conventionnel).

C'est donc le déploiement de la politique de marchandisation de la santé qui, parce qu'elle se développe sur un marché imparfait, accroît le nombre d'actes gratuits. La fréquence de ces actes serait alors d'autant plus sensible que le praticien acquière un jugement marchand de son activité et, en corollaire, la possibilité de moduler ses honoraires.

Le second paradoxe peut s'énoncer ainsi : ce sont les acteurs les plus soucieux des formes collectives d'exercice de la médecine libérale qui véhiculent massivement les instruments les plus pointus de la politique marchande de santé. Alors que les praticiens du secteur 2 augmentent le nombre d'actes gratuits, l'échantillon, étudié dans son entier, se caractérise par une diminution déclarée du nombre d'actes gratuits, tous motifs confondus, associée à l'augmentation du nombre et du montant des dépassements d'honoraires. Si les motivations invoquées pour expliquer ces évolutions sont multiples, la volonté d'inciter le patient à respecter certains comportements ou de le sanctionner domine en nombre. Or ce mécanisme d'incitation/sanction reproduit la logique incitative issue du référentiel marchand de politique publique. Ainsi, en instrumentalisant leurs tarifs, les secteurs $1 \mathrm{C}$ et $1 \mathrm{FH}$, les plus nombreux, stimulent le calcul rationnel et individuel des patients.

$\mathrm{Au}$ total, les comportements des médecins en termes d'actes gratuits et de modulation des tarifs mettent en évidence trois comportements différents qu'il est possible d'analyser au prisme de trois des espaces discursifs développés dans les conventions médicales. Si la fracture classique entre discours libéral traditionnel et discours libéral renouvelé se retrouve dans les choix initiaux des médecins, cette frontière se déplace, voire disparaît avec l'immixtion d'un discours marchand et d'une politique associée. Paradoxalement, cette politique mène les généralistes du secteur à honoraires libres à pratiquer, à l'encontre des principes libéraux, plus d'actes gratuits que ne le font les praticiens du secteur conventionné. Le discours marchand, en prônant la modulation des prix conduit aussi les mêmes médecins à élaborer leur propre « politique de santé » fondée sur leur propre jugement de la justice sociale et sur le seul critère libéral traditionnel de la qualité des soins.

À l'opposé, les praticiens les plus contraints dans leurs choix (secteur 1FM) et, à ce titre, les plus à même d'appliquer la politique de santé, sont les plus prompts à diminuer le nombre d'acte gratuits et à appliquer, tels que prévus dans les textes, les différents dépassements d'honoraires. Ces praticiens alimentent ainsi le paradoxe de la politique de santé. Entre les deux catégories précédentes, les praticiens des secteurs $1 \mathrm{C}$ et $1 \mathrm{FH}$, satisfaits d'exercer en secteur conventionné pour des motifs politiques et sociaux diminuent progressivement le nombre d'actes gratuits qu'ils effectuent, en cohérence avec la politique de santé d'une part et le principe libéral renouvelé qu'ils soutiennent d'autre part. Mais ces médecins se voient aussi instrumentaliser les actes gratuits et les dépassements d'honoraires pour distribuer des sanctions et/ou des bonus à leurs patients. 


\section{Conclusion}

L'analyse statistique et lexicométrique menée sur cet échantillon de 596 praticiens d'Îlede-France et de Bourgogne a mis en évidence des pratiques tarifaires spécifiques selon les groupes de praticiens. Ainsi, les omnipraticiens du secteur à honoraires libres sont-ils plus enclins à rendre gratuits certains actes (notamment des actes de prévention). À l'opposé, les praticiens n'ayant pas choisi d'exercer en secteur 1 sont les moins nombreux à pratiquer des actes gratuits.

L'analyse lexicométrique globale a montré l'opposition, dans le questionnaire, entre discours libéral traditionnel, principalement porté par les médecins du secteur à honoraires libres, et discours libéral renouvelé, porté par les praticiens du secteur à honoraires conventionnés ayant volontairement opté pour ce secteur. Cette opposition, est arbitrée par le rôle plus ou moins marqué du discours marchand. L'introduction du discours et des pratiques marchandes par la politique de santé s'accompagne en effet d'outils et de concepts auxquels les praticiens ne sont pas habitués. Au cœur de ceux-ci, le problème de l'évaluation de certains actes médicaux comme celui des actes non médicaux réalisés par les médecins a des effets paradoxaux.

Les résultats souffrent cependant de plusieurs défauts inhérents à ce type d'étude et aux choix effectués dans le traitement. En premier lieu, aucun redressement de l'échantillon n'a pu techniquement ${ }^{13}$ être réalisé alors que des écarts significatifs sont apparus entre les données statistiques de l'échantillon et les moyennes habituellement observées. Les femmes sont ainsi sous-représentées, les praticiens du secteur 2 sont surreprésentés, la durée de consultation est surévaluée. En second lieu, le caractère déclaratif des questions mène nécessairement à un risque de biais de déclaration. Celui-ci est d'autant plus important que les questions sont ouvertes et que les questionnaires ont été remplis directement par les omnipraticiens. Habituellement gênant, ce biais constitue sans doute un atout dans le cas de ce questionnaire. De nombreux médecins ont en effet exagéré ou schématisé leurs propos et facilité ainsi la classification du discours. Il n'en reste pas moins qu'en l'absence de population de référence, l'ampleur de cet effet d'amplification et, en conséquence, l'impact du biais de déclaration sur les résultats reste difficilement évaluable.

Sous ces limitations, l'hypothèse posée au départ de ce travail considérant que la politique économique de santé se reflète sur les comportements tarifaires des praticiens semble confirmée par l'analyse statistique et lexicométrique. Le discours médical, notamment celui des médecins interrogés, est profondément influencé par les représentations. Il convient toutefois de noter que les mécanismes de transmission ne sont pas encore totalement dévoilés. Ces mécanismes font l'objet d'hypothèses additionnelles, telle que celle considérant le tarif conventionnel comme une borne tarifaire inférieure en deçà de laquelle les praticiens pratiqueraient un acte gratuit, qui, pour être étayées, devraient faire l'objet d'analyses économétriques plus poussées.

\footnotetext{
${ }^{13}$ En raison du nombre de variables sur lesquelles le redressement était nécessaire et des corrélations connues entre ces variables d'une part. Compte tenu de la nature modale de plusieurs variables telles que l'âge ou la durée de consultation, qui empêchaient tout rapprochement avec les variables numériques habituellement utilisées d'autre part.
} 


\section{Bibliographique}

ABALLÉA P., BARTOLI F., ESCLOUS L. et YENI I., (2007), Les dépassements d'honoraires médicaux, IGAS, Paris.

ABECASSIS P. et DOMIN J.-P. (2009), L'évolution des conventions médicales à travers leur discours. Les maux de la politique économique de santé in Barnay T. et Legendre F. (Éds), Emploi et politiques sociales. Tome 1 : défis et avenirs de la protection sociale, Éditions de L'Harmattan, Paris, 71-84.

ABECASSIS P. et DOMIN J.-P., (2008), L'analyse textuelle avec Prospéro : comment parlent les conventions médicales in Batifoulier P., Gadreau M. et Vacarie I. (Éds), La gouvernance de l'assurance maladie : l'orientation marchande et ses paradoxes, DREES-MIRE, Paris, 78-103.

ALBOUY V. et DÉPREZ M., (2009), Mode de rémunération des médecins, Économie et prévision 188, 131-139.

ATTAL-JOUBERT K. et VANDERSCHELDEN M., (2009), La démographie médicale à l'horizon 2030 : de nouvelles projections nationales et régionales, Études et Résultats 679.

AUDRIC S., (2004), L'exercice en groupe des médecins libéraux, Études et Résultats 314.

AULAGNIER M., VIDEAU Y., COMBES J.-B., SEBBAH R., PARAPONARIS A., VERGER P. et VENTELOU B., (2007), Pratiques des médecins généralistes en matière de prévention : les enseignements d'un panel de médecins généralistes en Provence-Alpes-Côte d'Azur, Pratiques et organisations des soins 38 (4), 259-268.

BACHIMONT J., COGNEAU J. et LETOURMY A., (2006), Pourquoi les médecins généralistes n'observent-ils pas les recommandations de bonnes pratiques cliniques ? L'exemple du diabète de type 2, Sciences Sociales et Santé 24 (2), 75-103.

BATIFOULIER P., DOMIN J.-P. et GADREAU M., (2007a), Chapitre introductif. Politique de santé : la grande transformation, Économie appliquée 60 (1), 5-36.

BATIFOULIER P., DOMIN J.-P. et GADREAU M. (2007b), La gouvernance de l'assurance maladie au risque d'un État social marchand, Économie appliquée 60 (1), 101-126.

BATIFOULIER P. et GADREAU M., (2006a), Comportement du médecin et politique économique de santé. Quelle rationalité pour quelle éthique ?, Journal d'Économie Médicale 24 (5), 229-239.

BATIFOULIER P. et GADREAU M., (2006b), Régulation et coordination du système de santé. Des institutions invisibles à la politique économique in Eymard-Duvernay F. (Éd), L'économie des conventions, méthodes et résultats, La Découverte, Paris, 453-468.

BATIFOULIER P., GADREAU M. et LIEVAUT J. (2009), La décision tarifaire : des actes gratuits aux actes chers. Les effets pervers de la politique publique, Journal d'Économie Médicale 27 (4) 222-234.

BATIFOULIER P., GADREAU M. et VACARIE I., (2008), La gouvernance de l'assurance maladie : l'orientation marchande et ses paradoxes, DREES-MIRE, Paris.

BATIFOULIER P. et VENTELOU B., (2003), L'érosion de la part gratuite en médecine libérale. Discours économique et prophéties auto réalisatrices, Revue du MAUSS 21, 313-329.

BATIFOULIER P., VENTELOU B. et PROTOPESCU C., (2008), La coloration marchande des comportements médicaux. Une illustration entre la relation entre tarif et durée de consultation in Batifoulier P., Maryse G. et Vacarie I. (Éds), La gouvernance de l'assurance maladie : l'orientation marchande et ses paradoxes, DREES-MIRE, Paris, 108-122.

BÉJEAN S., PEYRON C. et URBINELLI R., (2002), Comportement et activité des médecins libéraux : une approche conventionnaliste, DREES-MIRE, Paris.

BLOY G., (2005), Savoirs professionnels en médecine générale : le cas du stage chez le praticien, Revue Française des Affaires Sociales 59 (1),103-125.

BLOY G., (2004), Socialisation et transmission professionnelles en médecine générale : étude sociologique du stage chez le praticien, DREES-MIRE, Paris.

BLOY G., (2002), Motivations et représentations de l'acte médical en médecine générale de ville : un regard sociologique in Béjean S. et Peyron C. (Éds), Santé, règles et rationalité, Economica, Paris, 73-110.

BOLTANSKI L. et CHIAPELLO É., (1999), Le nouvel esprit du capitalisme, Gallimard, Paris. 
BOLTANSKI L. et THÉVENOT L., (1991), De la justification. Les économies de la grandeur, Gallimard, Paris. BORIES-MASKULOVA V., (2008), Démographie et honoraires des médecins libéraux en 2006, Points de repère 23 .

BREUIL-GENIER P. et GOFFETTE C., (2006), La durée des séances des médecins généralistes, Études et Résultats 481.

DARRINÉ S. et NIEL X., (2001), Les médecins omnipraticiens au 1er janvier 2000, Études et Résultats 99.

DIDRY C., (1998), Les comités d'entreprise face aux licenciements collectifs, trois registres d'argumentation, Revue française de sociologie 39 (3), 495-534.

DOMIN J.-P., (2003), Crise sociale et institutionnalisation des solidarités : une application au système de santé (1890-1940) in Guillaume P. (Éd), Les solidarités (2). Du terroir à l'État., Éditions de la MSH d'Aquitaine, Bordeaux, 73-93.

FAURE A., POLLET G. et WARIN P., (1995), La construction du sens dans les politiques publiques débats autour de la notion de référentiel, Ed. l'Harmattan, Paris.

GRIMALDI A. et VALLANCIEN G. (2010), Les paradoxes du médecin à l'hôpital, Les Tribunes de la santé 26 (1), 21-37.

HALL P. A., (1993), Policy Paradigms, Social Learning and the State, Comparative Politics 25 (3), 275-296.

HASSENTEUFEL P. et SMITH A., (2002), Essoufflement ou second souffle ? L'analyse des politiques publiques à la française, Revue française de science politique 52 (1), 53-73.

HATZFELD H., (1963), Le grand tournant de la médecine libérale, Éditions ouvrières, Paris.

HERZLICH C., BUNGENER M.,PAICHELER G., ROUSIN P. et ZUBER M. C.,(1993), Cinquante ans d'exercice de la médecine en France : carrières et pratiques des médecins français (1930-1980), INSERM-Doin, Paris,.

JANUS K., AMELUNG V. E., BAKER L. C., GAITANIDES M., SCHWARTZ F. W. et RUNDALL T. G., (2008), Job Satisfaction and Motivation among Physicians in Academic Medical Centers: Insights from a Cross-National Study, Journal of Health Politics Policy and Law 33 (6),1133-1167.

JAUNAIT A., (2005), Comment pense l'institution médicale? Une analyse des codes français de déontologie médicale, Dalloz, Paris.

LABARTHE G., (2004), Les consultations et visites des médecins généralistes. Un essai de typologie, Études et Résultats 315.

LECA J., MULLER P., MAJONE G., THOENIG J.-C. et DURAN P., (1996), Enjeux, controverses et tendances de l'analyse des politiques publiques, Revue française de science politique 46 (1), 96-133.

LEGENDRE N., (2006), Les revenus libéraux des médecins en 2003 et 2004, Études et Résultats 457.

MULLER P., (2003), Les politiques publiques, PUF, Paris.

MULLER P., (2000), L'analyse cognitive des politiques publiques : vers une sociologie politique de l'action publique», Revue française de science politique 50 (2), 189-207.

ONDPS, (2006), Le rapport 2005. Synthèse générale, La Documentation française, Paris.

PALIER B., (2008), De la demande à l'offre, les réformes de la protection sociale en France in GuillemarD A.-M. (Éd), Où va la protection sociale, PUF, Paris, 119-138.

PALIER B., (2005), Gouverner la sécurité sociale. Les réformes du système français de protection sociale depuis 1945, PUF, Paris.

PIERRU F., (2007), Hippocrate malade de ses réformes, Éditions du Croquant, Bellecombe-en-Bauges.

REINERT M., (2007), Posture énonciative et mondes lexicaux stabilisés en analyse statistique de discours, Langage et société 121-122, 189-202.

ROBELET M.,(2005), Réforme du système de santé et mobilisation éthique des médecins : un regard sociologique sur l'éthique des professions in Batifoulier P. et Gadreau M. (Éds), Éthique médicale et politique de santé, Economica, , Paris, 145-165. 
ROCHAIX L., (1997), Asymétries d'information et incertitude en santé : les apports de la théorie des contrats, Économie et Prévision 129-130, 11-24.

STEFFEN M., (1987), Les médecins et l'État en France, Politiques et management public 5 (3), 19-39.

THÉRET B., (2007), Les transformations de l'État social français depuis 1975 : une analyse centrée sur les politiques de santé et menée au regard des standards typologiques et théoriques du changement institutionnel, Économie appliquée 60 (1), 69-100.

VENTELOU B., PARAPONARIS A., SEBBAH R., AULAGNIER M., PROTOPESCU C., GOURHEUX J.-C. et VERGER P., (2005), Un observatoire des pratiques en médecine générale : l'expérience menée en PACA, Revue Française des Affaires Sociales 59 (1), 127-160.

VIDEAU Y., VENTELOU B., COMBES J.-B., VERGER P. et PARAPONARIS A., (2007), Les médecins généralistes face aux réformes. Les réactions du panel de médecins libéraux de la région PACA, Médecinel Sciences 23 (5), 538-544. 


\section{Annexe}

Composition du questionnaire (variables retenues pour l'étude)

\begin{tabular}{|c|c|c|}
\hline Type & Questions & Modalités (après traitement) \\
\hline $\mathrm{M}^{*}$ & 1. Vous êtes (homme/femme) & Femme; Homme \\
\hline $\mathrm{CM}$ & 2. Vous êtes âgé(e) de & 30 à 45 ans; 46 à $50 ; 51$ à $55 ; 56$ ans et + \\
\hline $\mathrm{CM}$ & 3. Vous êtes installé depuis : & 0 à 15 ans; 16 à 25 ans; + de 25 ans \\
\hline M & $\begin{array}{l}\text { 4. Le cabinet dans lequel vous exercez } \\
\text { aujourd'hui est situé: }\end{array}$ & $\begin{array}{l}\text { Quartier urbain résidentiel; urbain inter- } \\
\text { médiaire; urbain populaire; milieu semi- } \\
\text { rural ; rural }\end{array}$ \\
\hline $\mathrm{O}$ & \multicolumn{2}{|c|}{$\begin{array}{l}\text { 5. Pouvez-vous caractériser rapidement votre patientèle (âge, sexe, origine sociale, } \\
\text { types de demandes...)? }\end{array}$} \\
\hline $\mathrm{CM}$ & $\begin{array}{l}\text { 5. À combien estimez-vous la proportion } \\
\text { de patients bénéficiaires de la CMU dans } \\
\text { votre patientèle? }\end{array}$ & $\begin{array}{l}\text { - de } 5 \% \text { de la clientèle; de } 5 \% \text { à - de } 10 \% \text {; } \\
\text { de } 10 \% \text { à - de } 20 \% ;+ \text { de } 20 \% \text {; ne sais pas. }\end{array}$ \\
\hline $\mathrm{CM}$ & $\begin{array}{l}\text { 7. Chaque jour, combien estimez-vous faire } \\
\text { en moyenne de consultation: }\end{array}$ & $\begin{array}{l}\text { - de } 15 \text { consultations par jour; de } 14 \text { à } 19 ; \\
\text { de } 20 \text { à } 24 ; \text { de } 25 \text { à } 29 ;+ \text { de } 30\end{array}$ \\
\hline $\mathrm{CM}$ & $\begin{array}{l}\text { 8. Chaque jour, combien estimez-vous faire } \\
\text { en moyenne de visites : }\end{array}$ & $\begin{array}{l}\text { aucune visite par jour ; } 1 ; 2 ; 3 \text { à } 4 ; 5 \\
\text { visites et }+\end{array}$ \\
\hline $\mathrm{CM}$ & $\begin{array}{l}\text { 9. Pouvez-vous estimer la durée habituelle } \\
\text { d'une consultation à votre cabinet? }\end{array}$ & $\begin{array}{l}\text {-de } 15 \mathrm{mn} \text { par consultation; de } 15 \text { à - de } \\
20 \mathrm{mn} \text {; de } 15 \text { à - de } 30 \mathrm{mn} ;+ \text { de } 30 \mathrm{mn}\end{array}$ \\
\hline $\mathrm{O}$ & \multicolumn{2}{|c|}{$\begin{array}{l}\text { 10. Il vous arrive d'avoir affaire au médecin-conseil local de l'assurance-maladie. } \\
\text { Que pensez-vous globalement de cette relation? }\end{array}$} \\
\hline $\mathrm{O}$ & \multicolumn{2}{|c|}{ 11. Diriez-vous qu'elle a évolué ces dernières années? } \\
\hline M & 12. Avez-vous été médecin référent? & Non; Oui \\
\hline $\mathrm{O}$ & \multicolumn{2}{|l|}{ 13. Que pensiez-vous de ce dispositif? } \\
\hline $\mathrm{O}$ & \multicolumn{2}{|c|}{ 14. Que pensez-vous de la récente réforme du médecin traitant? } \\
\hline M & $\begin{array}{l}\text { 15. Dans quel secteur tarifaire de la conven- } \\
\text { tion médicale exercez-vous? }\end{array}$ & Exercice en secteur 1 ; en secteur 2 \\
\hline M & $\begin{array}{l}\text { 16. Avez-vous eu la possibilité de passer } \\
\text { en secteur } 2 \text { ? }\end{array}$ & $\begin{array}{l}\text { Oui et c'est fait; oui mais je ne l'ai pas } \\
\text { fait; non }\end{array}$ \\
\hline $\mathrm{O}$ & \multicolumn{2}{|c|}{ 17. Vous souvenez-vous de vos motivations de l'époque? D'hésitations? } \\
\hline $\mathrm{O}$ & \multicolumn{2}{|c|}{ 18. Pensez-vous avoir perdu des patients de ce fait (passer au secteur 2)? } \\
\hline $\mathrm{O}$ & \multicolumn{2}{|c|}{$\begin{array}{l}\text { 19. Les patients sont réputés adopter de plus en plus des comportements de consom- } \\
\text { mateurs. Cela se manifeste-t-il dans votre expérience? Comment? }\end{array}$} \\
\hline $\mathrm{O}$ & \multicolumn{2}{|c|}{$\begin{array}{l}\text { 20. Arrive-t-il que des patients commentent les honoraires que vous demandez? En } \\
\text { quels termes? Comment réagissez-vous? }\end{array}$} \\
\hline
\end{tabular}




\begin{tabular}{|c|c|c|}
\hline $\mathrm{CM}$ & $\begin{array}{l}\text { 21. Aujourd'hui vos honoraires sont habi- } \\
\text { tuellement de pour une consultation sans } \\
\text { RDV : }\end{array}$ & $\begin{array}{l}\text { TC; } \text { - de } 10 \% \text { au-dessus du TC; de } 10 \% \\
\text { à -de } 25 \% \text { du TC; de } 25 \% \text { à - de } 50 \% \text { du } \\
\text { TC } ;+ \text { de } 50 \% \text { du TC }\end{array}$ \\
\hline $\mathrm{O}$ & \multicolumn{2}{|c|}{$\begin{array}{l}\text { 22. Pouvez-vous indiquer avec quelle fréquence et dans quelles circonstances vous } \\
\text { faites varier vos tarifs? }\end{array}$} \\
\hline M & $\begin{array}{l}\text { 23. Êtes-vous amené à réaliser des actes } \\
\text { gratuits? }\end{array}$ & Non; Oui \\
\hline M & $\begin{array}{l}\text { 24. Si vous n'effectuez pas d'actes gratuits, } \\
\text { en avez-vous fait dans le passé? }\end{array}$ & Non; Oui \\
\hline $\mathrm{O}$ & \multicolumn{2}{|l|}{ 25. Pour quelle(s) raisons? } \\
\hline $\mathrm{CM}$ & $\begin{array}{l}\text { 26. Si vous effectuez des actes gratuits, } \\
\text { combien estimez-vous en avoir fait dans } \\
\text { le mois écoulé? }\end{array}$ & $\begin{array}{l}\text { - de } 5 \text { actes gratuits au cours du mois } \\
\text { dernier; de } 5 \text { à } 9 ; \text { de } 10 \text { à } 14 ; \text { de } 15 \text { à } \\
24 ; 25 \text { et }+\end{array}$ \\
\hline $\mathrm{O}$ & \multicolumn{2}{|c|}{ 27. Pour quels motifs [Effectuez-vous des actes gratuits]? } \\
\hline M & $\begin{array}{l}\text { 28. Avez-vous l'impression de faire plus } \\
\text { ou moins fréquemment [d'actes gratuits] } \\
\text { ces dernières années? }\end{array}$ & inchangé; + fréquemment; - fréquemment \\
\hline $\mathrm{O}$ & \multicolumn{2}{|c|}{$\begin{array}{l}\text { 29. Quelles sont aujourd'hui les principales raisons de votre appartenance au secteur } \\
\text { dans lequel vous exercez? }\end{array}$} \\
\hline $\mathrm{O}$ & \multicolumn{2}{|c|}{$\begin{array}{l}\text { 30. Que pensez-vous du fait que certains médecins de secteur } 1 \text { pratiquent des dépas- } \\
\text { sements sans autorisation? }\end{array}$} \\
\hline $\mathrm{O}$ & \multicolumn{2}{|c|}{$\begin{array}{l}\text { 31. Si vous exercez dans un cabinet de groupe, tous vos associés ont-ils les mêmes } \\
\text { pratiques que vous en matière d'honoraires? Cela peut-il être une source de tensions? }\end{array}$} \\
\hline M & $\begin{array}{l}\text { 32. Sentez-vous une concurrence entre } \\
\text { médecins dans votre secteur géographique }\end{array}$ & Non; Oui \\
\hline $\mathrm{O}$ & \multicolumn{2}{|c|}{$\begin{array}{l}\text { 33. Si vous sentez-vous une concurrence entre médecins dans votre secteur, comment } \\
\text { cela se manifeste-t-il? }\end{array}$} \\
\hline M & $\begin{array}{l}\text { 34. Avez-vous l'impression d'en pratiquer } \\
\text { plus ou moins fréquemment ces dernières } \\
\text { années? }\end{array}$ & -fréquemment; inchangé; + fréquemment \\
\hline $\mathrm{O}$ & \multicolumn{2}{|c|}{$\begin{array}{l}\text { 35. Que pensez-vous de la possibilité de dépassement en cas d'appel en visite sans } \\
\text { motif médical valable? }\end{array}$} \\
\hline $\mathrm{O}$ & \multicolumn{2}{|c|}{$\begin{array}{l}\text { 36. Comment utilisez-vous personnellement la possibilité de dépassement en cas } \\
\text { d'appel en visite sans motif médical valable? }\end{array}$} \\
\hline M & $\begin{array}{l}\text { 37. Pourriez-vous envisager de devenir } \\
\text { salarié de la sécurité sociale dans les années } \\
\text { qui viennent? }\end{array}$ & Non; Oui \\
\hline $\mathrm{O}$ & \multicolumn{2}{|l|}{ 38. À quelles conditions? } \\
\hline $\mathrm{O}$ & \multicolumn{2}{|c|}{$\begin{array}{l}\text { 39. Qu'est-ce qui aujourd'hui vous satisfait le plus dans votre exercice de la médecine } \\
\text { générale? }\end{array}$} \\
\hline
\end{tabular}




\begin{tabular}{|c|c|c|}
\hline $\mathrm{O}$ & \multicolumn{2}{|c|}{ 40. Qu'est-ce qui vous frustre ou vous inquiète le plus dans cet exercice? } \\
\hline M & $\begin{array}{l}\text { 41. Conseilleriez-vous l'exercice de la } \\
\text { médecine générale à un de vos enfants } \\
\text { attiré par la médecine? }\end{array}$ & Non; Oui \\
\hline $\mathrm{O}$ & \multicolumn{2}{|c|}{$\begin{array}{l}\text { 42. Pour quelles raisons Conseilleriez-vous (ou non) l'exercice de la médecine générale } \\
\text { à un de vos enfants attiré par la médecine? }\end{array}$} \\
\hline $\mathrm{O}$ & \multicolumn{2}{|c|}{$\begin{array}{l}\text { 44. Quelles seraient, à votre avis, les mesures susceptibles de rendre l'exercice libéral } \\
\text { de la médecine générale attirant pour les jeunes? }\end{array}$} \\
\hline $\mathrm{O}$ & \multicolumn{2}{|c|}{$\begin{array}{l}\text { 45. Si vous aviez demain les pleins pouvoirs pour réorganiser notre « système de } \\
\text { santé » (assurance-maladie, rémunération et coordination des professionnels, qualité } \\
\text { des soins,...) quelles seraient vos priorités? }\end{array}$} \\
\hline M & $\begin{array}{l}\text { 46. Dans quelle tranche se situent vos } \\
\text { revenus professionnels mensuels (incluant } \\
\text { toutes vos activités médicales, après déduc- } \\
\text { tion des charges et avant impôts): }\end{array}$ & $\begin{array}{l}- \text { de } 2000 € ; \text { de } 2000 \text { à }- \text { de } 3500 € ; \text { de } \\
3500 \text { à }- \text { de } 5000 € ; \text { de } 5000 \text { à }- \text { de } 6500 \\
€ ; \text { de } 6500 \text { à }- \text { de } 8000 € ; 8000 € \text { et+ }\end{array}$ \\
\hline $\mathrm{CM}$ & $\begin{array}{l}\text { 47. Acceptez-vous de vous situer politique- } \\
\text { ment [de } 0 \text { (gauche) à } 10 \text { (droite)] }\end{array}$ & $\begin{array}{l}\text { de } 0 \text { à }- \text { de } 2 ; \text { de } 2 \text { à }- \text { de } 4 ; \text { de } 4 \text { à - de } 6 ; \\
\text { de } 6 \text { à - de } 8 ; \text { de } 8 \text { à -de } 10 ; 10 \text { et }+\end{array}$ \\
\hline $\mathrm{O}$ & \multicolumn{2}{|c|}{ 48. Souhaitez-vous ajouter un commentaire libre? } \\
\hline
\end{tabular}

* $\boldsymbol{M}$ : variable modale; $\boldsymbol{C M}$; variable continue modalisée; $\boldsymbol{O}$ : variable textuelle (question ouverte. 


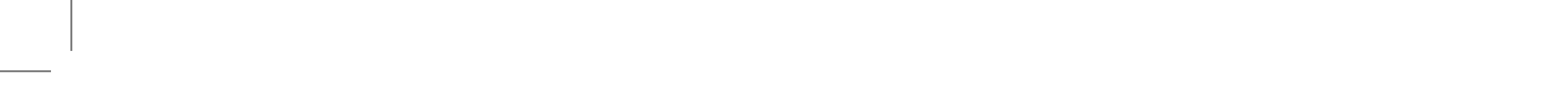

